\title{
Andrographolide Protects against Aortic Banding-Induced Experimental Cardiac Hypertrophy by Inhibiting MAPKs Signaling
}

\section{OPEN ACCESS}

Edited by:

Nicolau Beckmann,

Novartis Institutes for BioMedical

Research, United States

Reviewed by:

W. S. Fred Wong,

National University of Singapore,

Singapore

Mitchell K. P. Lai,

National University of Singapore,

Singapore

Rafael Agustín Burgos,

Universidad Austral de Chile, Chile

*Correspondence:

Wei Deng

vivideng1982@whu.edu.cn

Specialty section:

This article was submitted to Cardiovascular and Smooth Muscle

Pharmacology,

a section of the journal

Frontiers in Pharmacology

Received: 22 July 2017 Accepted: 26 October 2017 Published: 14 November 2017

Citation:

Wu QQ, Ni J, Zhang N, Liao HH,

Tang QZ and Deng W (2017)

Andrographolide Protects against Aortic Banding-Induced Experimental Cardiac Hypertrophy by Inhibiting MAPKS Signaling.

Front. Pharmacol. 8:808. doi: 10.3389/fphar.2017.00808

\author{
Qing Q. Wu ${ }^{1,2,3}$, Jian Ni ${ }^{1,2,3}$, Ning Zhang ${ }^{1,2,3}$, Hai H. Liao ${ }^{1,2,3}$, Qi Z. Tang ${ }^{1,2,3}$ and \\ Wei Deng ${ }^{1,2,3,4 *}$
}

${ }^{1}$ Department of Cardiology, Renmin Hospital of Wuhan University, Wuhan, China, ${ }^{2}$ Cardiovascular Research Institute, Wuhan University, Wuhan, China, ${ }^{3}$ Hubei Key Laboratory of Cardiology, Wuhan, China, ${ }^{4}$ Department of Cardiology, The Fifth Affiliated Hospital of Xinjiang Medical University, Ürümqi, China

Despite therapeutic advances, heart failure-related mortality rates remain high. Therefore, understanding the pathophysiological mechanisms involved in the remodeling process is crucial for the development of new therapeutic strategies. Andrographolide (Andr), a botanical compound, has potent cardio-protective effects due to its ability to inhibit mitogen-activated protein kinases (MAPKs). Andr has also been shown to inhibit inflammation and apoptosis, which are factors related to cardiac hypertrophy. Our aim was to evaluate the effects of Andr on cardiac hypertrophy and MAPKs activation. Thus, mice were subjected to aortic banding (AB) with/without Andr administration (25 mg/kg/day, orally). Cardiac function was accessed by echocardiography and hemodynamic parameters. Our results showed that Andr administration for 7 weeks decreased cardiac dysfunction and attenuated cardiac hypertrophy and fibrosis in $A B$ mice. Andr treatment induced a strong reduction in the transcription of both hypertrophy (ANP, BNP, and $\beta-\mathrm{MHC}$ ) and fibrosis related genes (collagen I, collagen III, CTGF, and TGF $\beta$ ). In addition, cardiomyocytes treated with Andr showed a reduced hypertrophic response to angiotensin II. Andr significantly inhibited MAPKs activation in both mouse hearts and cardiomyocytes. Treatment with a combination of MAPKs activators abolished the protective effects of Andr in cardiomyocytes. Furthermore, we found that Andr also inhibited the activation of cardiac fibroblasts via the MAPKs pathway, which was confirmed by the application of MAPKs inhibitors. In conclusion, Andr was found to confer a protective effect against experimental cardiac hypertrophy in mice, suggesting its potential as a novel therapeutic drug for pathological cardiac hypertrophy.

Keywords: andrographolide, cardiac hypertrophy, cardiomyocyte, fibroblast, MAPKs

\footnotetext{
Abbreviations: Andr, andrographolide; ANP, atrial natriuretic peptide; AT1R, Ang II type 1 receptor; BNP, brain natriuretic peptide; CTGF, connective tissue growth factor; $\mathrm{dP} / \mathrm{dt}$ max, maximal rate of pressure development; $\mathrm{dP} / \mathrm{dt}$ min, minimal rate of pressure decay; ERK, extracellular signal-regulated kinase; GAPDH, glyceraldehyde-3-phosphate dehydrogenase; H\&E, hematoxylin and eosin; HO-1, heme oxygenase 1; HR, heart rate; LV, left ventricle; LVEDd, LV end-diastolic diameter; LVESd, LV end-systolic diameter; LVPWd, left ventricular posterior wall thickness; MAPK, mitogen-activated protein kinase signaling; MHC, myosin heavy chain; PSR, picrosirius red; TGF $\beta$, transforming growth factor $\beta$; $\alpha$-SMA, $\alpha$-smooth muscle actin.
} 


\section{INTRODUCTION}

Heart failure (HF) is a burgeoning problem that affects more than 20 million individuals worldwide (Tham et al., 2015). Cardiac hypertrophy is a response of the heart to increased workload (such as aortic stenosis, hypertension, and dilated cardiomyopathy) and various insults (such as myocarditis and myocardial infarction). It typically progresses to heart failure (Lyon et al., 2015; Shimizu and Minamino, 2016). Increased cardiomyocyte size and thickening of ventricular walls are the main features of cardiac hypertrophy (Lyon et al., 2015; Shimizu and Minamino, 2016). Maladaptive hypertrophy involves increased cardiomyocyte hypertrophy and, apoptosis as well as increased fibroblast activation, which are associated with reduced systolic function and increased heart stiffness (Samak et al., 2016; Shimizu and Minamino, 2016). Left ventricular hypertrophy is positively correlated with an increased risk of adverse cardiovascular events (Hill and Olson, 2008). Accumulating evidence indicates that multiple signaling pathways participate in the progression of cardiac hypertrophy, such as insulin growth factor/PI3K/Akt, protein kinase $\mathrm{C}$, and mitogen-activated protein kinases (MAPKs), $\beta$-adrenergic receptor, calcineurin/NFAT, and $\mathrm{Ca}^{2+} /$ CaMKII signaling (Hou and Kang, 2012; Tham et al., 2015). However, the complexity of the mechanism underlying the transition from hypertrophic processes to heart failure and the difficulty in reversing cardiac hypertrophy contribute to the high mortality rates of heart failure. Therefore, new pharmacological agents that selectively inhibit the progression of cardiac hypertrophy are of great therapeutic interest.

MAPKs are involved in diverse biological events, including proliferation, differentiation, metabolism, motility, survival, and apoptosis (Liu and Molkentin, 2016). MAPKs subfamilies include ERK1/2, c-Jun NH2-terminal kinases (JNK), and P38 kinase (Liu and Molkentin, 2016). ERK1/2 signaling co-ordinates the eccentric and concentric growth of the heart. In addition, ERK autophosphorylation at Thr 188 facilitates ERK1/2 activity toward nuclear targets, which is a critical event in the induction of ERKmediated cardiac hypertrophy in response to various stimuli (Lorenz et al., 2009; Li W.M. et al., 2017). JNK can shuttle between the cytoplasm and the nucleus to exert its effects. Overactivation of JNK leads to a hypertrophic phenotype, and abrogation of JNK activity attenuates endothelin-1 (ET-1) and the pressure overload-induced hypertrophic response (Li W.M. et al., 2017). P38 is rapidly activated within a few minutes of exposure to aortic pressure or volume overload. Cardiac-specific overexpression of P38 leads to enhanced cardiac hypertrophy in response to pressure overload (Liao et al., 2001). The complexity of the signaling transduction network makes it impossible and imprudent to label any molecule as definitively 'bad' or 'good.' Thus, by focusing on network interactions rather than individual signaling molecules, we have a better chance of influencing the outcome.

Andrographis paniculata is a traditional medicinal herb that is used in China (Banerjee et al., 2017). Andrographolide is the major bioactive component of Andrographis paniculata. To date, studies have reported many pharmacological effects of Andr, such as anti-inflammatory (Ren et al., 2016), anti-oxidant (Chen et al., 2014), antihyperglycemic (Li et al., 2015), and hepatoprotective properties (Pan et al., 2017). Recent studies have found that Andr inhibits aconitine-induced arrhythmia by inhibiting voltagegated $\mathrm{Na}^{+}\left(\mathrm{I}_{\mathrm{Na}}\right)$, and $\mathrm{Ca}^{2+}\left(\mathrm{I}_{\mathrm{CaL}}\right)$ (Zeng et al., 2017). By

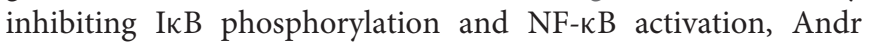
relieves lipopolysaccharide-induced cardiac malfunctions in mice (Zhang et al., 2015). Andr has also been reported to protect against hypoxia/reoxygenation injury in cardiomyocytes by regulating glutathione levels (Woo et al., 2008). Recently, Hsieh YL reported that Andrographis paniculata extract attenuates pathological cardiac hypertrophy and apoptosis in high-fat dietfed mice (Hsieh et al., 2016). All these findings suggest the cardiac protective effects of Andr. Andr was also reported to inhibit MAPKs in many disease models, including acute lung injury (Peng et al., 2016), rheumatoid arthritis (Li Z.Z. et al., 2017), Alzheimer's disease (Yang et al., 2017), and ischemic stroke models (Yen et al., 2016). These studies indicate that Andr may exert anti-hypertrophic effects by regulating MAPKs. Aortic banding $(\mathrm{AB})$ is a reliable model of left ventricular pressure overload for the study of the progression from compensated hypertrophy to heart failure, thus enabling the monitoring of cardiac remodeling (Martin et al., 2012). It is known that pressure overload can activate the renin-angiotensin system and induce the release of angiotensin II (Ang II), which activates the $\mathrm{G} \alpha$ (q) protein-coupled receptor signaling pathway (Wu et al., 2015). Thus, Ang II was used in vitro to induce cardiac hypertrophy in cardiomyocytes. The aim of our study was to explore the effects of Andr on pressure overload-induced cardiac hypertrophy, and fibrosis as well as the underlying mechanisms.

\section{MATERIALS AND METHODS}

\section{Chemicals}

Andrographolide was purchased from Shanghai Winberb Medical S\&T Development Co. Ltd. (Shanghai, China) with a purity $>98 \%$ as determinated by high-performance liquid chromatography analysis.

\section{Animals}

Eight to ten weeks old male C57/BL6 mice were purchased from the Institute of Laboratory Animal Science, CAMS\&PUMC (Beijing, China). Mice were housed in the Cardiovascular Research Institute of Wuhan University (Wuhan, China) with controlled temperature and humidity. $\mathrm{AB}$ surgery was performed as previously described (Wu et al., 2015, 2016). After 1 week of $A B$ or sham surgery, the animals were treated with $A$ dr daily $(25 \mathrm{mg} / \mathrm{kg}$ body weight/day, oral gavage, suspended in $0.5 \%$ carboxymethyl cellulose solution) until 8 weeks after surgery. Four groups were included: the vehicle-sham group (veh-sham, $n=15)$, the Andr-sham group $(n=15)$, the vehicle- $A B$ group (veh-AB, $n=15)$, and the Andr-AB group $(n=15)$. All the experimental procedures were in accordance with the institutional guidelines and approved by the Animal Care and 
TABLE 1 | Primer sequences for RT-PCR.

\begin{tabular}{|c|c|c|}
\hline mRNA & Forward & Reverse \\
\hline ANPa & ACCTGCTAGACCACCTGGAG & CCTTGGCTGTTATCTTCGGTACCGG \\
\hline BNP & GAGGTCACTCCTATCCTCTGG & GCCATाTCCTCCGACППTCTC \\
\hline$\beta-\mathrm{MHC}^{\mathrm{a}}$ & CCGAGTCCCAGGTCAACAA & CTTCACGGGCACCCTTGGA \\
\hline Collagen $1^{a}$ & AGGCTTCAGTGGTITGGATG & CACCAACAGCACCATCGTTA \\
\hline Collagen \|\|$^{\mathrm{a}}$ & AAGGCTGCAAGATGGATGCT & GTGCTTACGTGGGACAGTCA \\
\hline CTGF $^{\mathrm{a}}$ & AGGGCCTCTTCTGCGATITC & CTITGGAAGGACTCACCGCT \\
\hline TGF $\beta^{a}$ & ATCCTGTCCAAACTAAGGCTCG & ACCTCTITAGCATAGTAGTCCGC \\
\hline $\mathrm{GAPDH}^{\mathrm{a}}$ & ACTCCACTCACGGCAAATTC & TCTCCATGGTGGTGAAGACA \\
\hline ANPb & AAAGCAAACTGAGGGCTCTGCTCG & TTCGGTACCGGAAGCTGTTG CA \\
\hline $\mathrm{BNPb}$ & CAGCAGCTTCTGCATCGTGGAT & TTCCTTAATCTGTCGCCGCTGG \\
\hline$\beta-\mathrm{MHC}^{\mathrm{b}}$ & TCTGGACAGCTCCCCATTCT & CAAGGCTAACCTGGAGAAGATG \\
\hline Collagen $\mathrm{I}^{\mathrm{b}}$ & GAGAGAGCATGACCGATGGATT & TGGACATTAGGCGCAGGAA \\
\hline Collagen $\| I^{\mathrm{b}}$ & AAGGGCAGGGAACAACTGAT & GTGAAGCAGGGTGAGAAGAAAC \\
\hline CTGF $^{b}$ & GGAAGACACATITGGCCCTG & GCAATITAGGCGTCCGGAT \\
\hline GAPDH ${ }^{b}$ & GACATGCCGCCTGGAGAAAC & AGCCCAGGATGCCCTITAGT \\
\hline
\end{tabular}

Sequences are listed 5'-3'. a Primers used in mouse experiments. b Primers used for H9c2 rat cardiomyocytes and neonatal rat cardiac fibroblasts.

Use Committee of Renmin Hospital of Wuhan University (Approval number: WHRM 2015 W01; exact date of approval: January 1st, 2015).

\section{Echocardiography}

Cardiac functions were measured in our laboratory ( $\mathrm{Wu}$ et al., 2015, 2016). Briefly, echocardiography was performed on anesthetized (1.5\% isoflurane) mice using a MyLab 30CV ultrasound system (Biosound Esaote, Genoa, Italy) with a 10$\mathrm{MHz}$ linear array ultrasound transducer.

Parasternal short axis images were obtained at the level of the mid-papillary muscle in M-mode. Left ventricular (LV) dimensions from five consecutive cardiac cycles were measured and averaged, including LVEDs, LVEDd, end-diastolic LVPWd, and end-systolic LVPW (LVPWs). Fractional shortening (FS) and LV ejection fraction (EF) were calculated using the LVEDs and LVEDd values.

\section{Measurement of Hemodynamic Parameters}

Hemodynamic parameters were measured in our laboratory ( $\mathrm{Wu}$ et al., 2015, 2016). Briefly, hemodynamics were measured in anesthetized ( $1.5 \%$ isoflurane) mice using cardiac catheterization. A microtip catheter transducer (SPR-839; Millar Instruments, Houston, TX, United States) was inserted into the right carotid artery and advanced into the LV. Data including HR, enddiastolic pressure (EDP), end-systolic pressure (ESP), dP/dt max, and $\mathrm{dP} / \mathrm{dt}$ min were analyzed.

\section{Histological Analysis}

Heart slides were obtained using previously described methods (Wu et al., 2015, 2016). HE staining was performed to assess the cardiomyocyte cross-sectional area (CSA) and to observe the morphology of striated muscle. Sirius red in saturated picric acid (PSR) staining was used to determine interstitial fibrosis. A quantitative digital image analysis system (Image-Pro Plus, version 6.0; Media Cybernetics, Rockville, MD, United States) was used to trace a single myocyte (100-200 myocytes in each group).

\section{Quantitative Real-Time Polymerase Chain Reaction (RT-PCR)}

Total RNA and cDNA were prepared previously described (Wu et al., 2015, 2016).

We performed 20- $\mu$ l reactions according to the manufacturer's protocol with the following cycling parameters: $95^{\circ} \mathrm{C}$ for $5 \mathrm{~min}$; 45 cycles of $95^{\circ} \mathrm{C}$ for $10 \mathrm{~s}, 60^{\circ} \mathrm{C}$ for $10 \mathrm{~s}$, and $72^{\circ} \mathrm{C}$ for $10 \mathrm{~s} ; 95^{\circ} \mathrm{C}$ for $5 \mathrm{~s} ; 60^{\circ} \mathrm{C}$ for $1 \mathrm{~min} ; 97^{\circ} \mathrm{C}$ for $0.11 \mathrm{~s}$; and $40^{\circ} \mathrm{C}$ for $10 \mathrm{~min}$. The results were analyzed with the $2^{-\Delta \Delta \mathrm{Ct}}$ method and normalized to GAPDH gene expression. The primer sequences used in the RT-PCR experiment are listed in Table 1.

\section{Western Blotting}

Total protein was extracted from heart tissue and cells. The Bicinchoninic Acid Protein Assay kit (Thermo Fisher Scientific, Inc., Waltham, MA, United States) was used to measure the concentration of protein. The protein lysates were separated by $10 \%$ SDS-PAGE and transferred to PVDF membranes as previously described (Wu et al., 2015, 2016). The next day, blots were incubated with secondary antibodies for $1 \mathrm{~h}$. A two-color infrared imaging system (Odyssey; LI-COR Biosciences, Lincoln, NE, United States) was used to scan the protein blots. The primary antibodies used in this study were as follows: phospho-ERK1/2 Thr202/Tyr204 [1:1000, \#4370, Cell Signaling Technology (CST), Danvers, MA, United States], ERK1/2 (1:1000, \#4695, CST), phospho-P38Thr180/Tyr182 (1:1000, \#4511, CST), P38 (1:1000, \#9212, CST), phosphorJNK1/2Thr183/Tyr185 (1:1000, \#4668, CST), JNK1/2 (1:1000, \#9285, CST), smad4 (1:1000, \#9515, CST), and GAPDH (1:1000, \#2118, CST). 


\section{Cell Culture}

H9c2 cells were prepared according to our laboratory's protocols (Wu et al., 2015). The H9c2 cardiomyocytes were obtained from the Cell Bank of the Chinese Academy of Sciences, in Shanghai, China and cultured in DMEM (C11995; GIBCO, Thermo Fisher, Waltham, MA, United States) supplemented with $10 \%$ fetal bovine serum (10099; GIBCO) in an atmosphere containing 5\% $\mathrm{CO}_{2}$ inside a humidified incubator (SANYO $18 \mathrm{M}$, Osaka, Japan) at $37^{\circ} \mathrm{C}$. The cells were divided into four groups: the control group (CON), the Ang II ( $1 \mu \mathrm{M}$, Sigma) treatment group, the Andr $(12.5,25$, or $50 \mu \mathrm{M})$ group, and the Ang+Andr $(12.5,25$, or $50 \mu \mathrm{M})$ group. After treatment for $24 \mathrm{~h}$, cells from six wells were harvested for PCR analysis, while cells from 24 wells were used for immunofluorescence staining.

\section{Isolation and Culture of Cardiac Fibroblasts}

Neonatal rat cardiac fibroblasts were prepared to our laboratory's protocols (Wu et al., 2017a). Briefly, neonatal rats that were born within 3 days were sacrificed, and the hearts were collected. The hearts were cut into $1-\mathrm{mm}^{3}$ tissue and digested in $0.125 \%$ trypsin for $15 \mathrm{~min}$ at $34^{\circ} \mathrm{C}$ for a total of five times. The digestive fluid was collected and centrifuged. Cells were resuspended, filtered and then seeded onto $100-\mathrm{mm}$ plates for $90 \mathrm{~min}$. After removing the cardiomyocytes, the cardiac fibroblasts were cultured in DMEM/F12 containing 10\% FBS at $37^{\circ} \mathrm{C}$ in a humidified incubator with $5 \% \mathrm{CO} 2$. Before treatment with Ang II $(1 \mu \mathrm{M})$ and $\operatorname{Andr}(12.5,25$, or $50 \mu \mathrm{M})$, the cells were cultured in $1 \%$ FBS for $12 \mathrm{~h}$. After treatment for $24 \mathrm{~h}$ cells from six wells were harvested for PCR analysis while cells from 24 wells were used for immunofluorescence staining.

\section{Cell Counting Kit-8 Assay}

Cell viability was evaluated using the cell counting kit (CCK)-8 assay, according to the manufacturer's instructions. Briefly, $10 \mu \mathrm{l}$ of CCK-8 solution was added to each well of a 96-well plate, and the absorbance was measured at $450 \mathrm{~nm}$ using an ELISA reader (Synergy HT, Bio-tek, Winooski, VT, United States) after a 4-h incubation. The effect of Andr on cell viability was expressed as the percentage of viable cells compared with that in the vehicle group, which was set at $100 \%$.

\section{Immunofluorescence}

Culture medium was discarded, and the cells were washed with PBS; then, 4\% paraformaldehyde was used to fix the cells for $15 \mathrm{~min}$ at room temperature. After rinsing with PBS, the cells were permeabilized with $0.5 \%$ Triton $\mathrm{X}-100$ and then rinsed with PBS three times for 5 min each. Primary antibodies against $\alpha$-actinin (Millipore, 2207266, Darmstadt, Germany) and $\alpha$-SMA (Abcam, ab7817) at a 1:100 dilution were added to H9c2 cells and cardiac fibroblasts, respectively, in a 24 -well plate at $4^{\circ} \mathrm{C}$ overnight. The next day, sections were washed with PBS and then incubated with Alexa Fluor ${ }^{\circledR} 488$ goat anti-mouse IgG $(\mathrm{H}+\mathrm{L})$ antibodies for $60 \mathrm{~min}$ at $37^{\circ} \mathrm{C}$. Finally, after washing with PBS, Slow Fade Gold antifade reagent with DAPI was used to seal the sections before observation and imaging with a fluorescence microscope. Image-Pro Plus 6.0 was used to analyze the images ( $n=5$ samples per group and $n=100+$ cells were analyzed per group).

\section{Statistical Analysis}

All values were presented as the mean \pm SEM. SPSS 19.0 for Windows was used for the analysis. One-way ANOVA followed by post hoc Tukey test was performed for the data analysis. Twoway ANOVA followed by post hoc Tukey test was performed to evaluate the time-dependent anti-hypertrophic effects of Andr in vitro. A $p$-value $<0.05$ (two-tailed) was considered statistically significant.

\section{RESULTS}

\section{Andr Improves Cardiac Function after Chronic Pressure Overload in Mice}

To evaluate the effect of Andr on cardiac function, echocardiography was performed 8 weeks after AB (Figure 1A). The results showed that wall thickness (LVPWs, LVPWd, Figure 1B), chamber dilation (LVEDd, Figure 1C), and cardiac function (increased LVEF and LVFS, Figure 1D) were in the Andr-AB group than in the veh-AB group. There was no significant difference in HR among groups (Figure 1E). In addition, hemodynamics showed that Andr treatment improved systolic function and diastolic function after $A B$ surgery (Figures 1F-H). No obvious differences were observed between the sham-vehicle and Andr mice.

\section{Andr Attenuates Cardiac Hypertrophy after Chronic Pressure Overload in Mice}

Eight weeks after AB, Andr-treated mice presented decreased heart weight, which was accessed by heart weight to body weight ratios and heart weight to tibia length ratios (Figures 2A,B). Andr-treated mice also exhibited a strikingly decreased heart size and decreased cardiomyocyte CSA compared to vehicle treated animals (Figures 2C,D). Consistently, the expression of hypertrophic genes was significantly decreased in Andr-treated mice after AB (Figure 2E). Thus, Andr remarkably protected the heart from pressure overload-induced cardiac hypertrophy and dysfunction.

\section{Andr Attenuated Cardiac Fibrosis after Chronic Pressure Overload in Mice}

Cardiac fibrosis, one of the main features of cardiac hypertrophy, was evaluated by PSR staining. After 8 weeks of AB, dramatic interstitial fibrosis was observed in all $\mathrm{AB}$ mice, but the extent of fibrosis was decreased in the hearts from the Andr$\mathrm{AB}$ group (Figures 3A,B). The expression of myocardial pro-fibrotic genes was also down-regulated by Andr treatment (Figure 3C). 

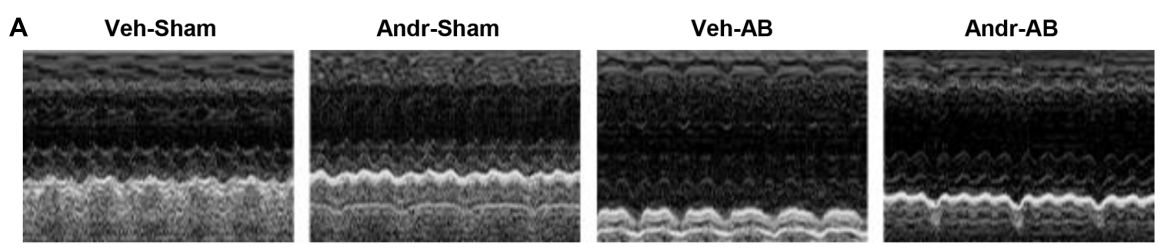

B
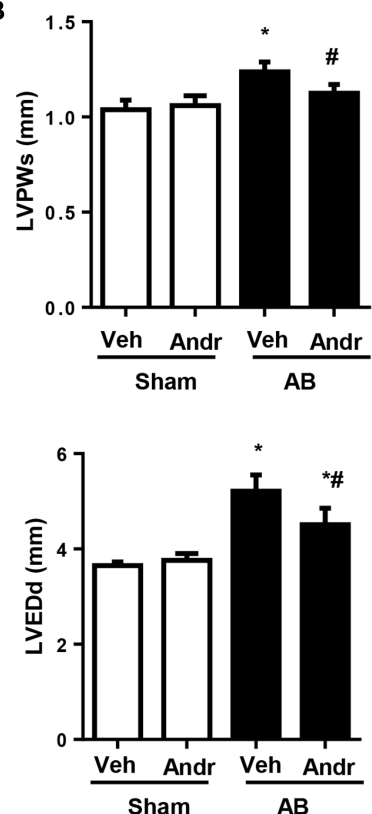

E
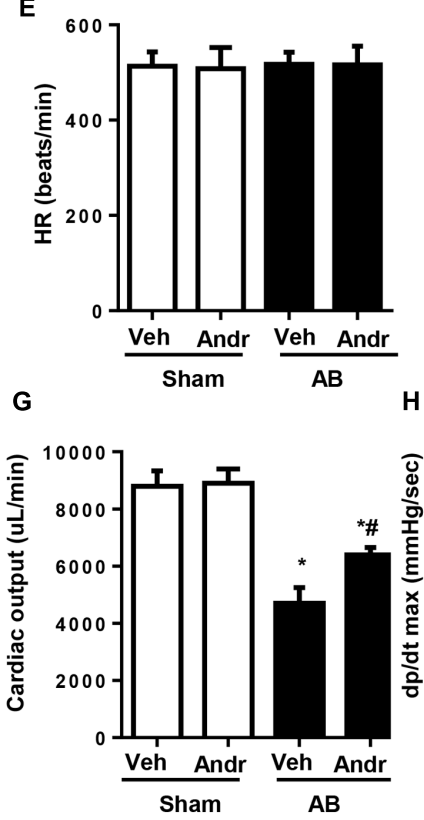

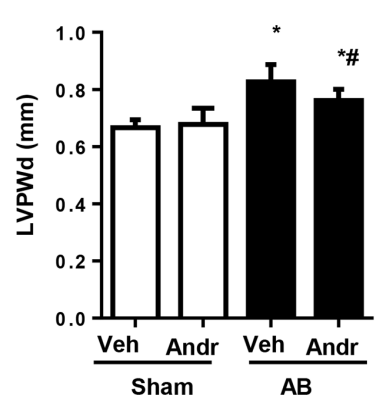

C
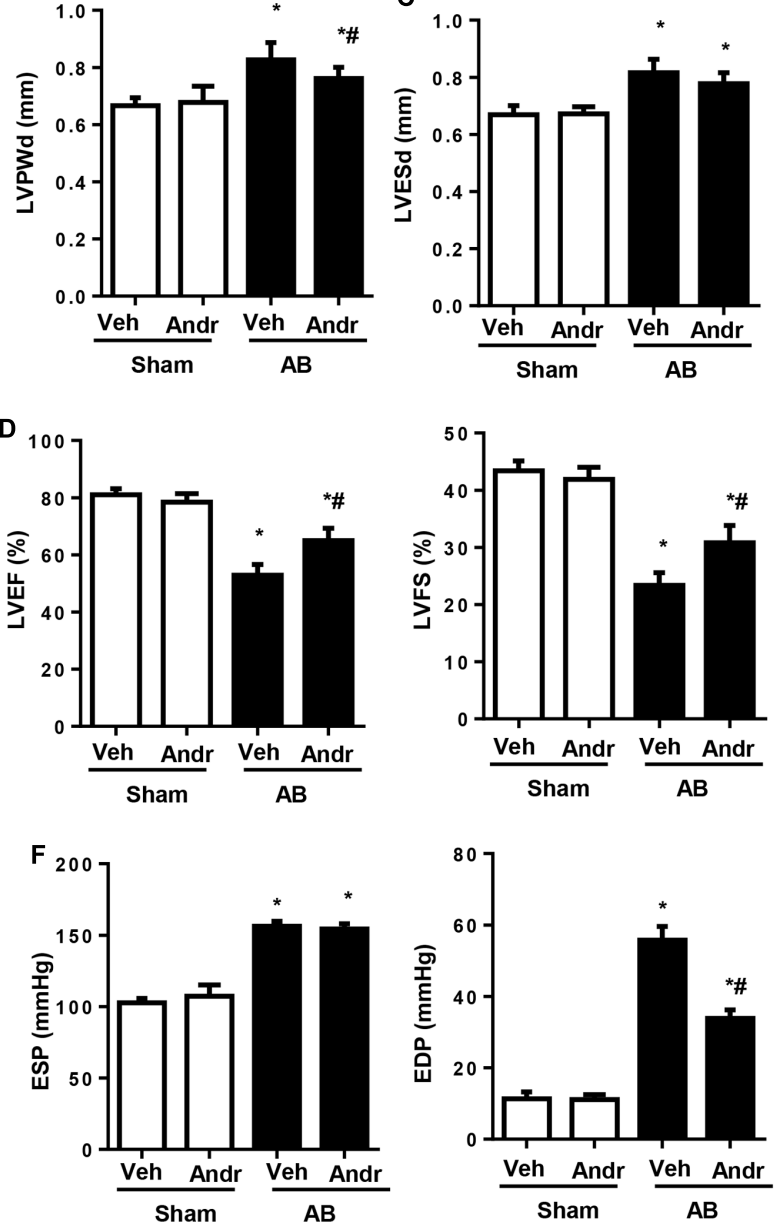

$$
\text { H }
$$

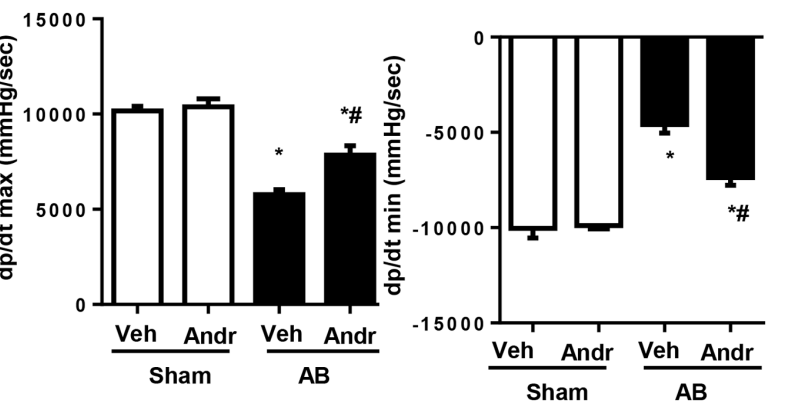

FIGURE 1 | Andr improves cardiac function after chronic pressure overload in mice. (A-D) Echocardiography was performed at the end of the study (8 weeks) $(n=8)$. (A) Representative images. Andr attenuated the AB-induced increase in wall thickness [B, left ventricular (LV) posterior wall thickness at the end of systole (LVPWs) and, LV posterior wall thickness at the end of diastole (LVPWd)], and LV diameter (C), including LVESd and LVEDd as well as attenuated AB-induced changes in ejection fraction (EF) and fractional shortening (FS) (D). (E-H). Hemodynamics analysis was performed at the end of the study (8 weeks) $(n=8)$. HR, heart rate; ESP, end-systolic pressure; EDP, end-diastolic pressure; CO, cardiac output; dP/dt max, maximal rate of pressure development; dP/dt min, minimal rate of pressure decay. ${ }^{*} P<0.05$ compared with the corresponding sham group. ${ }^{\#} P<0.05$ vs. the veh-AB group. AB, aortic banding. 

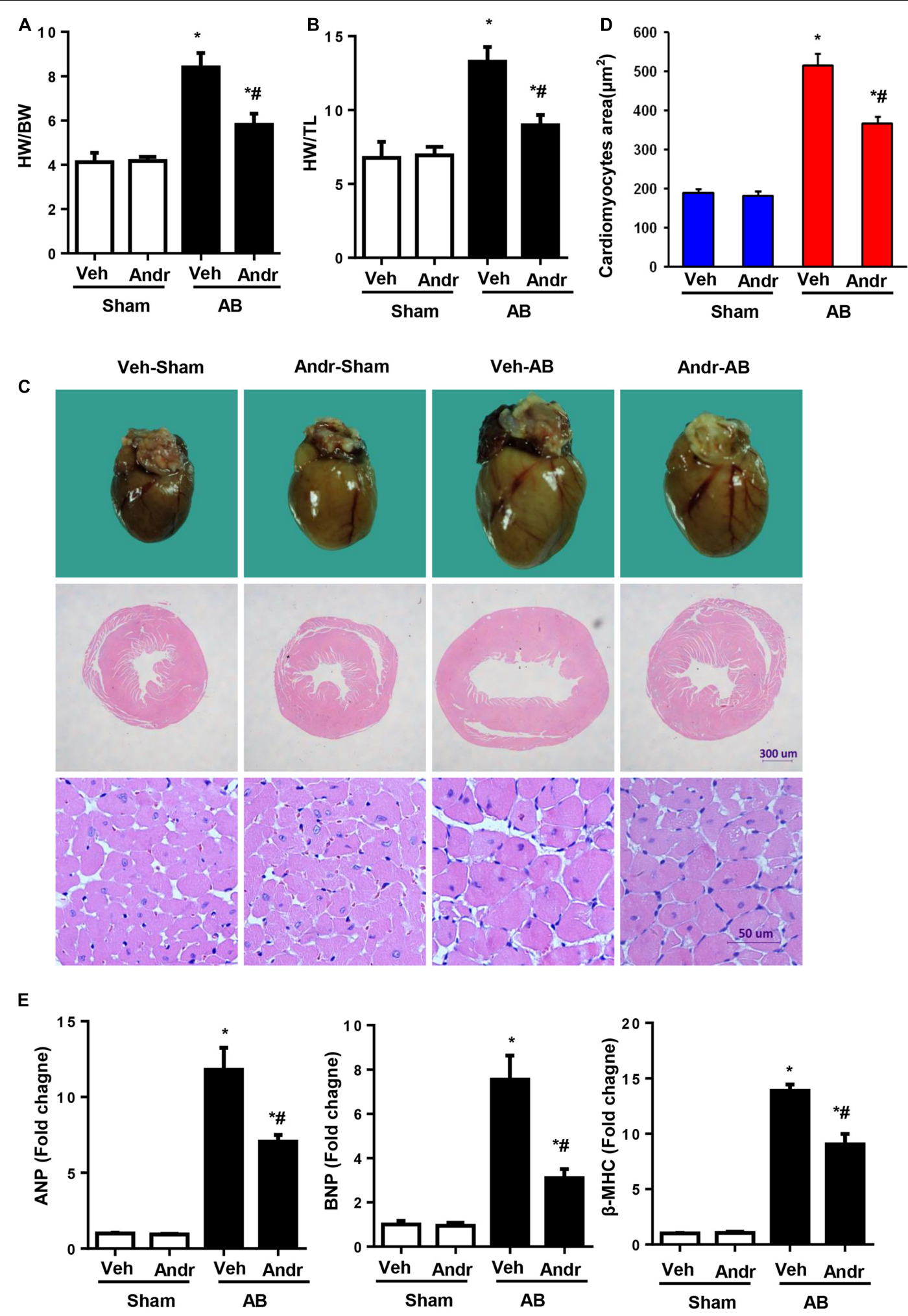

FIGURE 2 | Andr attenuates cardiac hypertrophy after chronic pressure overload in mice. (A,B) Statistical results of the heart weight/body weight (HW/BW) ratio, and heart weight/tibial length $(\mathrm{HW} / \mathrm{TL})$ ratio $(n=8)$. (C) Gross hearts, and HE staining of hearts from sham and aortic banding (AB) mice at 8 weeks post-surgery. (D) Myocyte cross-sectional areas (CSAs) of the indicated groups $(n=200+)$. (E) Expression of transcripts for ANP, BNP, and $\beta$-myosin heavy polypeptide (MHC) induced by $A B$ was determined by reverse transcription-polymerase chain reaction analysis $(n=6)$. The results are presented as a fold change, and the results are normalized to GAPDH gene expression. ${ }^{*} P<0.05$ as compared with the corresponding sham group. ${ }^{\#} P<0.05$ vs. the veh-AB group. AB, aortic banding. 

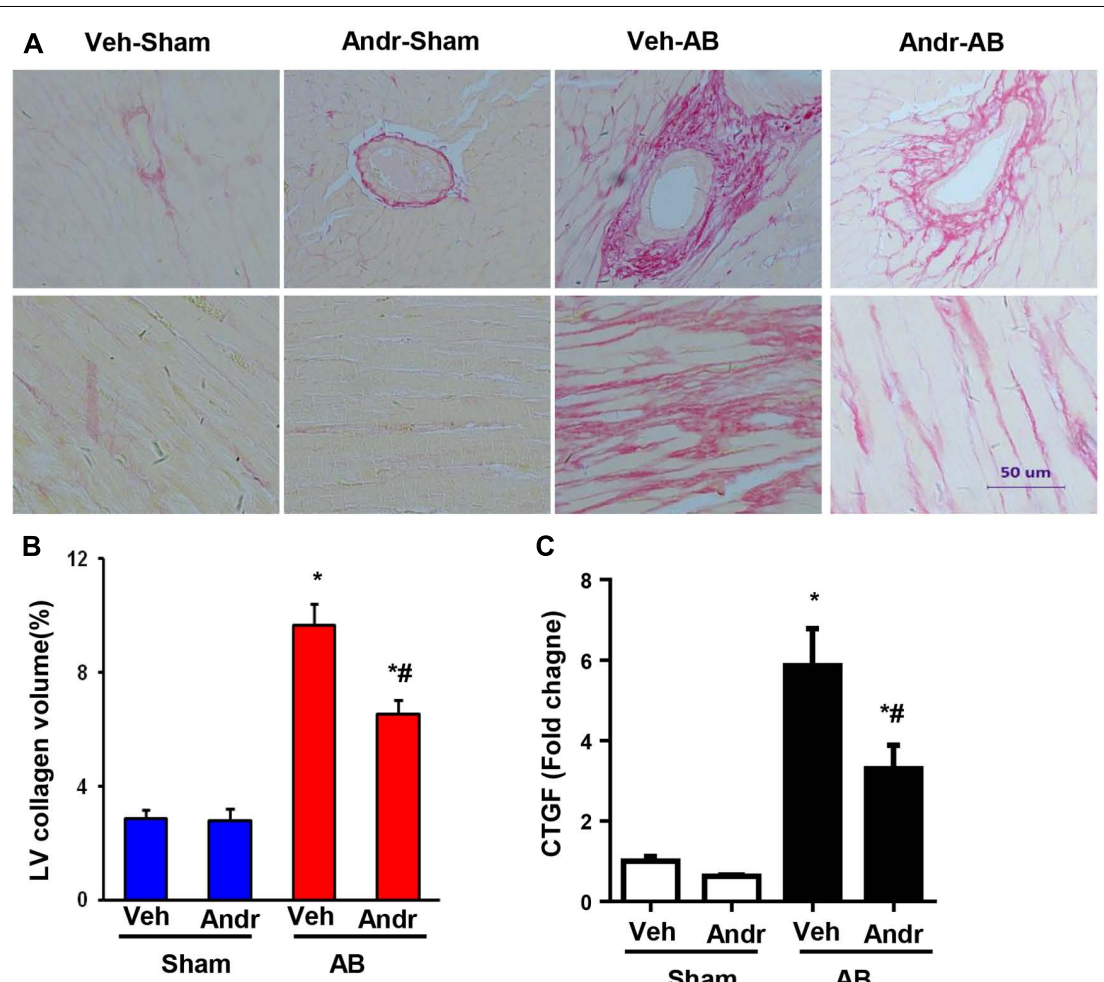

C
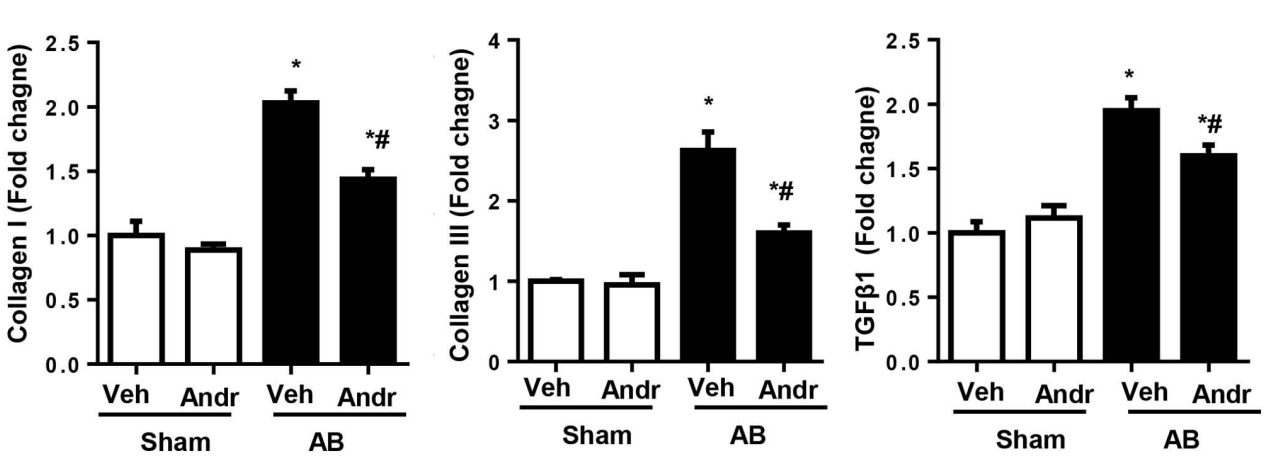

FIGURE 3 | Andr attenuated cardiac fibrosis after chronic pressure overload in mice. (A) Left ventricular histological sections from the indicated groups were stained with PSR $(n=6)$. (B) Fibrotic areas in the histological sections were quantified using an image-analysis system. (C) The mRNA expression of CTGF, collagen I, collagen III, fibronectin, and TGF $\beta 1$ in the myocardium was analyzed in the indicated groups using reverse transcription-polymerase chain reaction ( $n=6)$. The results are presented as a fold change, and the results are normalized to GAPDH gene expression. ${ }^{*} P<0.05$ compared with the corresponding sham group. ${ }^{\#} P<0.05$ vs. the veh-AB group.

\section{Andr Suppresses Ang II Induced Cardiomyocyte Hypertrophy}

To further determine whether Andr could attenuate cardiomyocyte hypertrophy, we treated cardiomyocytes with different concentrations of Andr $(0,12.5,25$, or $50 \mu \mathrm{M})$ and stimulated them with Ang II for $24 \mathrm{~h}$. The cell counting kit assay-8 assay revealed that $\operatorname{Andr}(12.5,25$, or $50 \mu \mathrm{M})$ treatment did not affect cardiomyocyte viability (Figure 4A). Compared with the Ang II group, Andr treatment dramatically blunted the prohypertrophic effect of Ang II in a dose-dependent manner as indicated by the cell surface area, and expression of fetal genes (Figures 4B-D). Thus, $50 \mu \mathrm{M}$ Andr was used in subsequent experiments.. After stimulating with Ang II for 6, 12, and 24 h, the cardiomyocytes exhibited gradually increased fetal gene expression, but Andr treatment nearly abolished this increase in the hypertrophic response (Figure $4 \mathrm{E}$ ). These results indicate an important anti-hypertrophic role for Andr in cardiomyocytes.

\section{Andr Blocks MAPKs Signaling in Vivo and in Vitro}

We further gained insight into the molecular events mediating the anti-hypertrophic effect of Andr. Andr has been reported to exert its anti-inflammatory (Ren et al., 2016), anti-oxidant (Chen et al., 2014), antihyperglycemic (Li et al., 2015), and hepatoprotective properties (Pan et al., 2017) by regulating MAPKs signaling. Thus, we detected the protein expression 

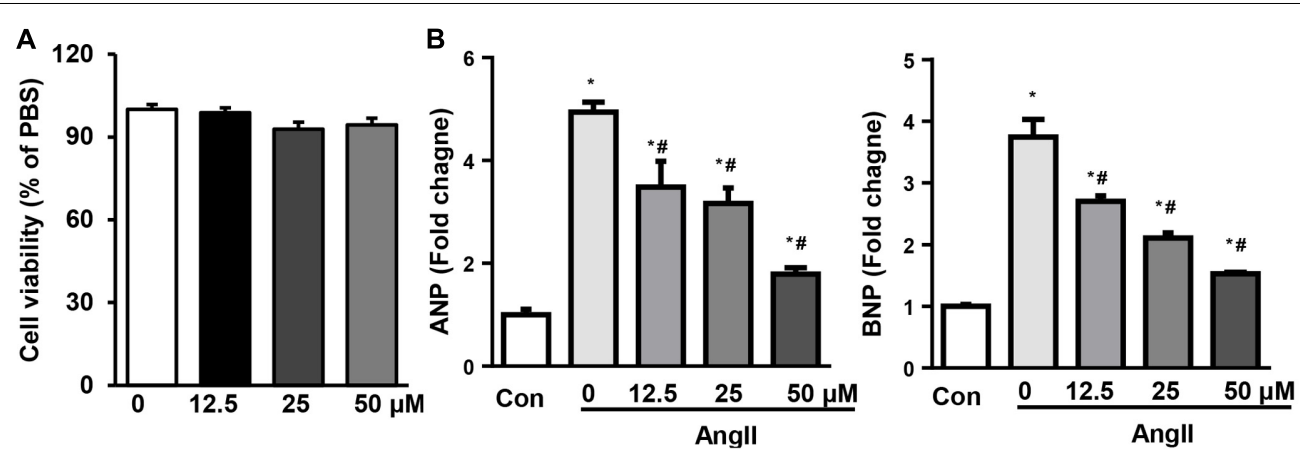

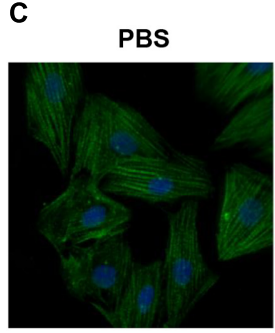

$25 \mu \mathrm{M}$
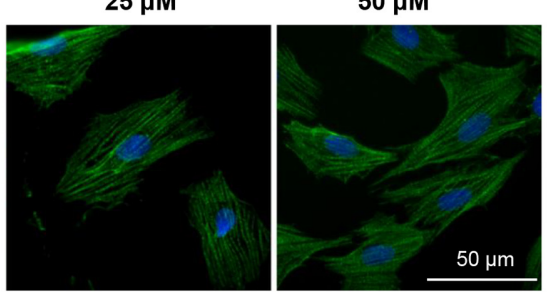

E
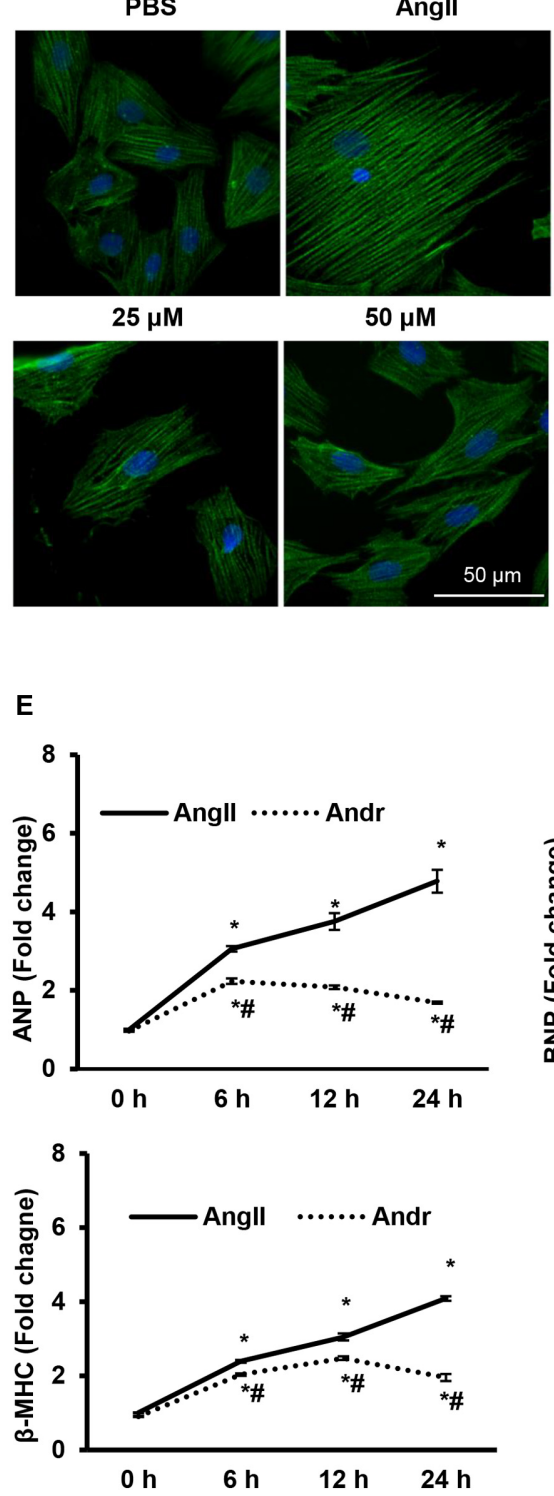

$12.5 \mu \mathrm{M}$

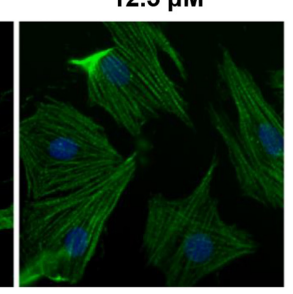

D
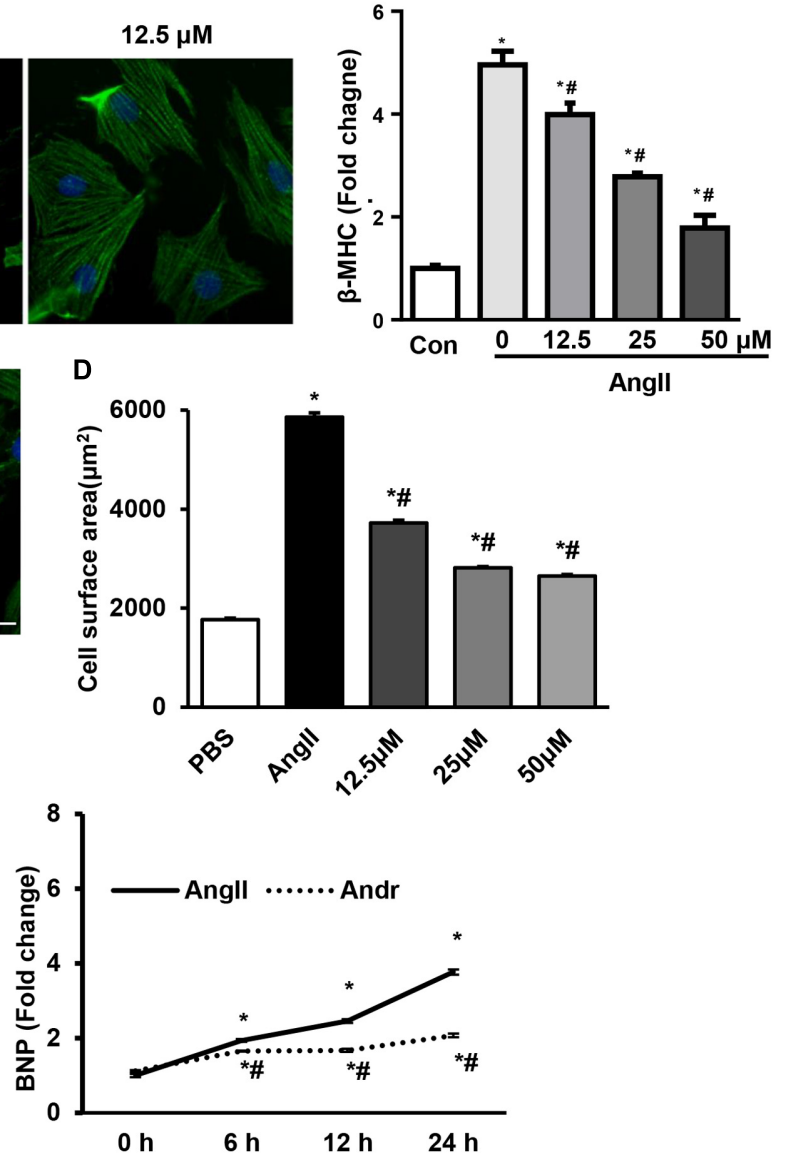

FIGURE 4 | Andr suppresses Ang II-induced cardiomyocyte hypertrophy. Cardiomyocytes were stimulated with Ang II (1 $\mu$ M) and treated with different concentrations of Andr (0, 12.5, 25, or $50 \mu \mathrm{M})$. (A) Cell viability was accessed by the cell counting kit- 8 assay $(n=5)$. (B) The mRNA levels of ANP, BNP, and $\beta-\mathrm{MHC}$ in cardiomyocytes in the indicated groups $(n=6)$. (C,D) Immunofluorescence staining of $\alpha$-actinin and the cell surface area of cardiomyocytes in the indicated groups ( $n=5$ samples, and $n=100+$ cells per group). ${ }^{*} P<0.05$ compared with the control group. \#P<0.05 vs. the Ang II group. (E) Cardiomyocytes were stimulated with Ang II (1 $\mu \mathrm{M})$ and treated with Andr $(50 \mu \mathrm{M})$ for 0, 6, 12, and $24 \mathrm{~h}$. The mRNA levels of ANP, BNP, and $\beta$-MHC in cardiomyocytes in the indicated groups $(n=6)$. The results are presented as a fold change, and the results are normalized to GAPDH gene expression. ${ }^{*} P<0.05$ compared with the corresponding 0 h group. ${ }^{\#} P<0.05$ vs. the corresponding Ang II group. 

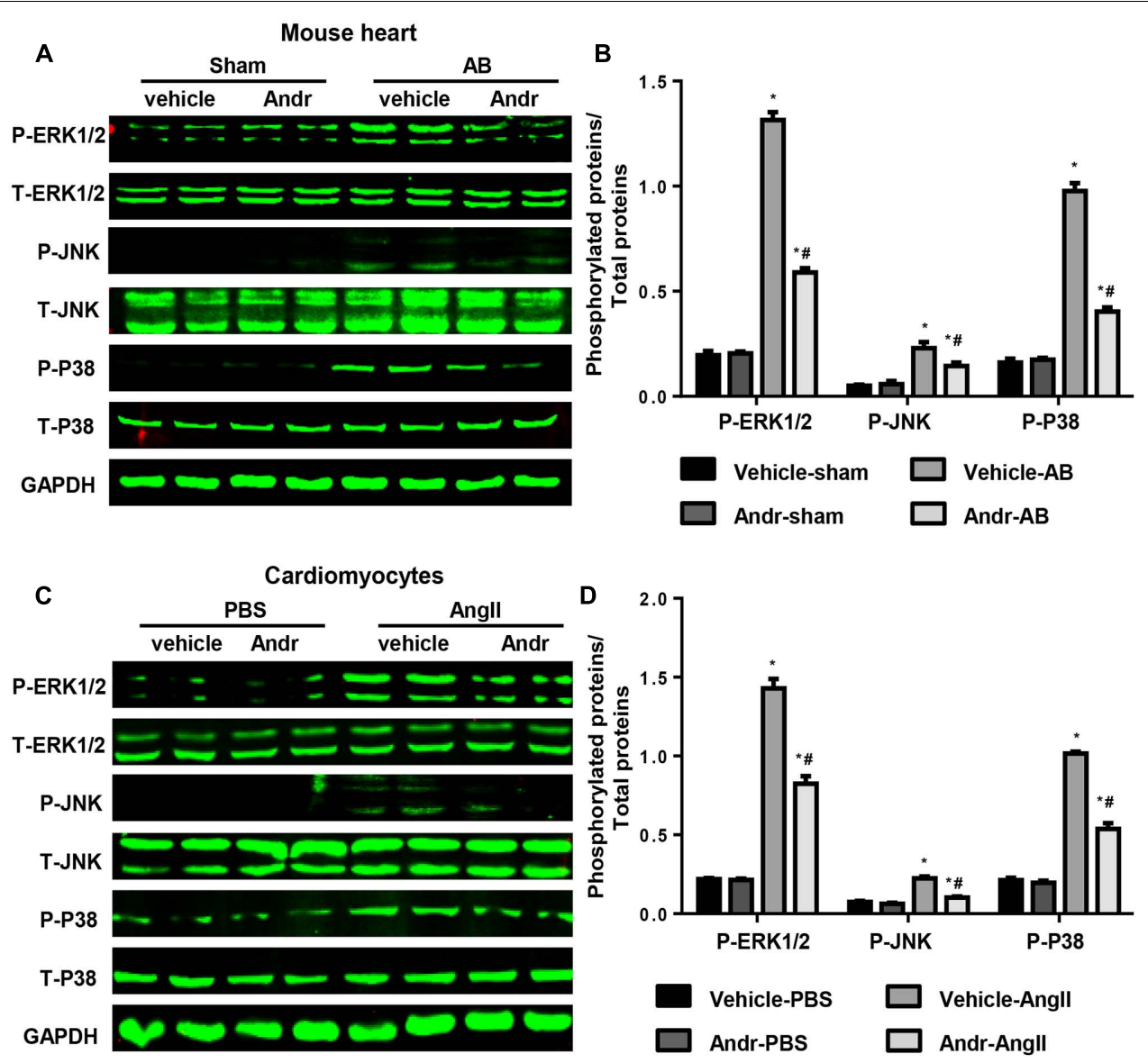

FIGURE 5 | Andr blocks MAPKs signaling in vivo and in vitro. (A) Representative blots of phosphorylated (P-) and total (T-) ERK1/2, JNK, and P38 in the heart tissues of mice in the indicated groups $(n=6)$. (B) Comparison of expression among the indicated groups. ${ }^{*} P<0.05$ compared with the corresponding sham group. $\# P<0.05$ vs. the veh-AB group. (C) Representative blots of phosphorylated (P-) and total (T-) ERK1/2, JNK, and P38 in the cardiomyocytes in the indicated groups $(n=6)$. (D) Comparison of expression among the indicated groups. ${ }^{*} P<0.05$ compared with the corresponding PBS group. ${ }^{*} P<0.05$ vs. the vehicle-Ang $\|$ group.

of MAKPs. We found that MAPKs activation, including ERK1/2, JNK, and P38, was induced by pressure overload, but was strongly down-regulated in Andr-treated mice after 8 weeks of $\mathrm{AB}$ (Figures $\mathbf{5 A}, \mathbf{B}$ ). In line with this in vivo data, treatment cardiomyocytes with Andr resulted in dephosphorylation of ERK1/2, JNK, and P38 after Ang II stimulation (Figures 5C,D). The results imply that MAPKs may mediate the anti-hypertrophic effects of Andr.

\section{Andr-Mediated Cardioprotection Depends on the Inhibition of MAPKs in Cardiomyocytes}

The effect of Andr on MAPKs was further confirmed by MAPKs inhibitors. Cardiomyocytes were treated with ERK1/2 inhibitor (SCH772984, $5 \mu \mathrm{M}$, Selleck), JNK inhibitor (SP600125, $10 \mu \mathrm{M}$, Sigma), and/or P38 inhibitor (SB209063, $10 \mu \mathrm{M}$, Medchem Express) as well as stimulated with Ang II. These inhibitors did not affect cardiomyocyte cell viability as shown in Figure 6A. None of these inhibitors could elicit a antihypertrophic response that was comparable to Andr when applied alone, but treatment with a combination of these three inhibitors did achieve a similar anti-hypertrophic response. Andr treatment could further augment the anti-hypertrophic effect of each inhibitor, as shown by the augmented reduction in surface area and fetal gene expression (Figures 6B-J). These findings suggest that suppression of all members of MAPKs (ERK1/2, JNK, and P38), underlies the anti-hypertrophic effects of Andr on cardiomyocytes.

\section{Andr Reduces Cardiac Fibroblast Activation via MAPKs}

Andr treatment ameliorated cardiac fibrosis at 8 weeks after $\mathrm{AB}$ in mice (shown in Figure 3). As cardiac fibroblasts play a major role in cardiac fibrosis, we then investigated whether Andr affects fibroblast activation. Cell counting kit- 8 assay revealed that Andr $(12.5,25$, or $50 \mu \mathrm{M})$ treatment did not affect fibroblast viability (Figure 7A). Ang II-induced fibroblast activation, proliferation, and function were inhibited by Andr treatment in a dosedependent manner (Figures 7A-D). Andr also inhibited Ang II-induced MAPKs activation (including ERK1/2, JNK, and 

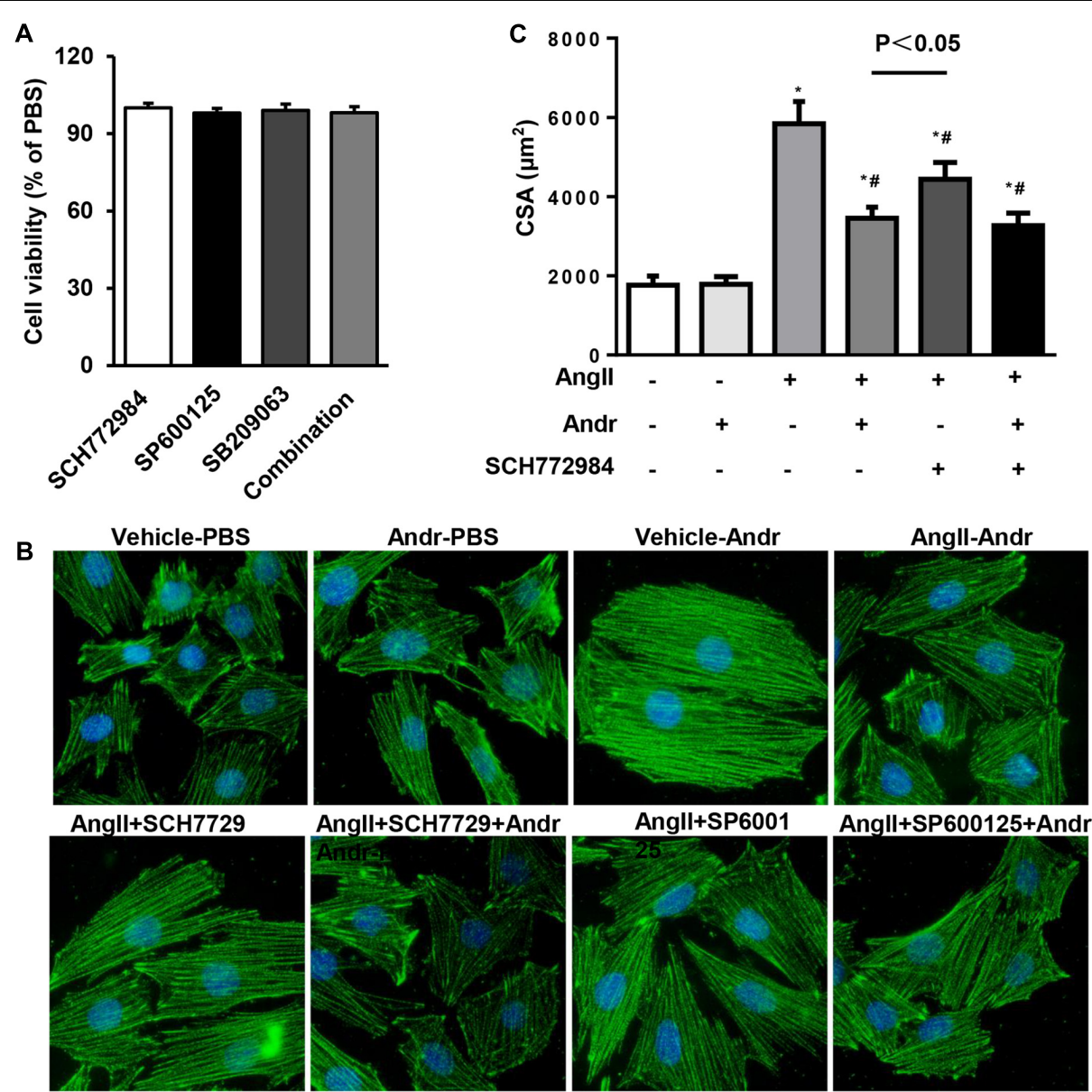

Angll+SCH7729+Andr

Angll+SP6001

Angll+SP600125+Andr
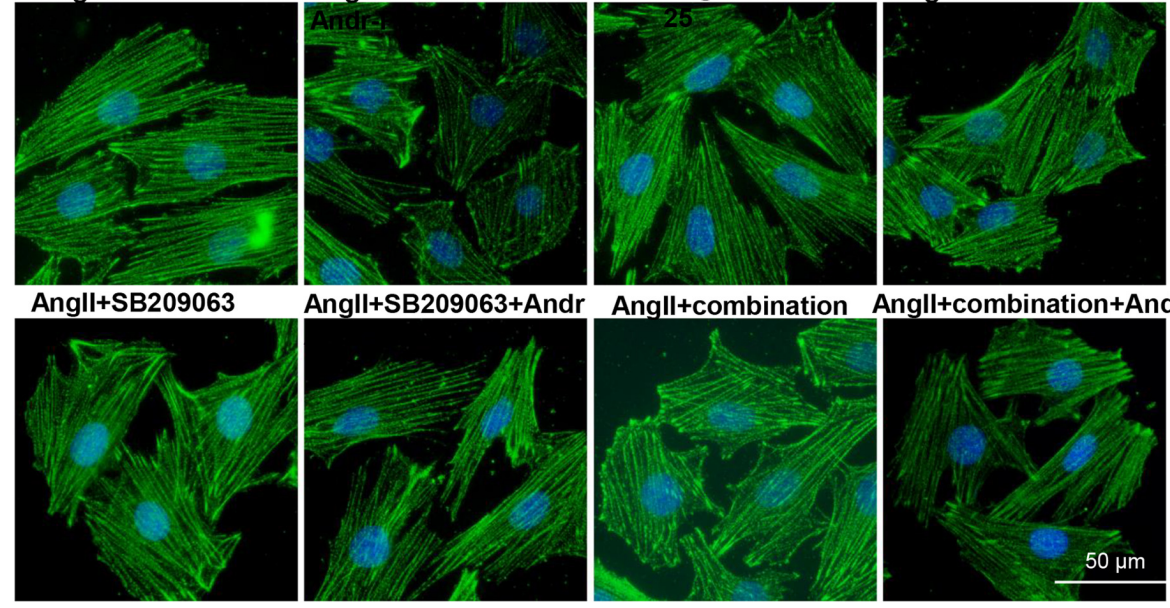

Angll+SB209063+Andr Angll+combination Angll+combination+Andr

D
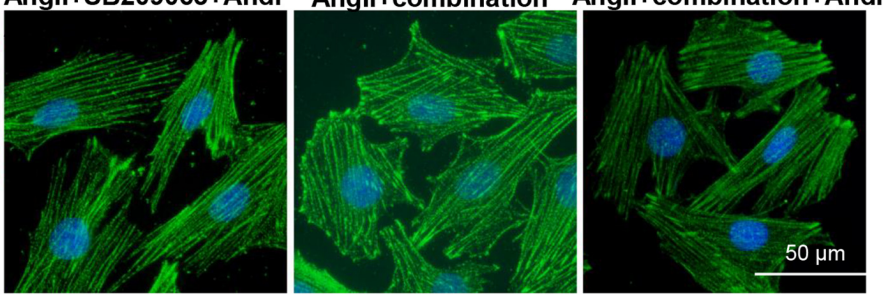

E
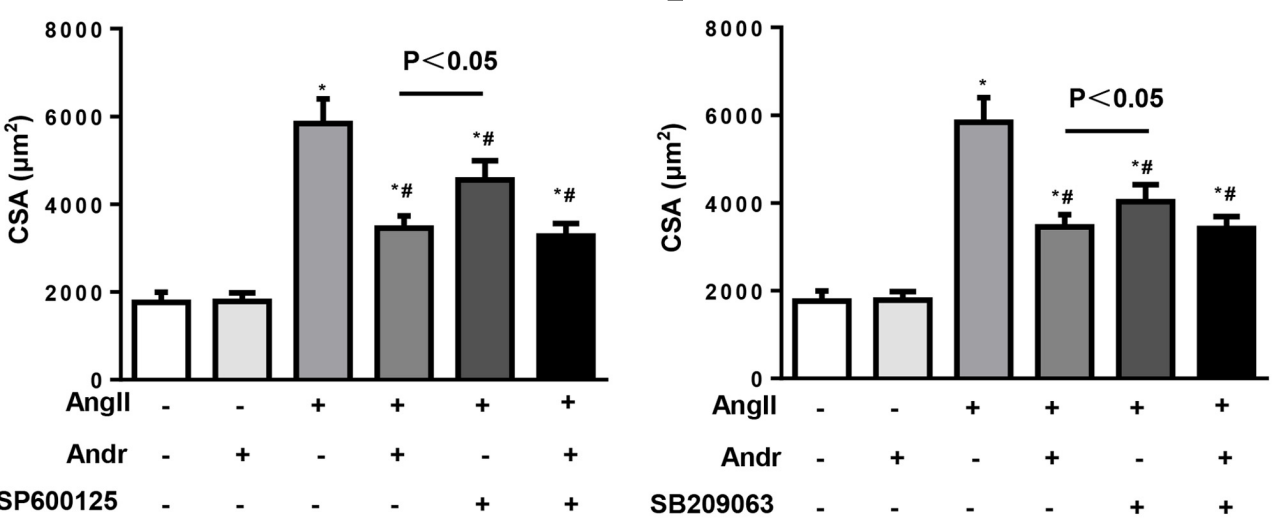

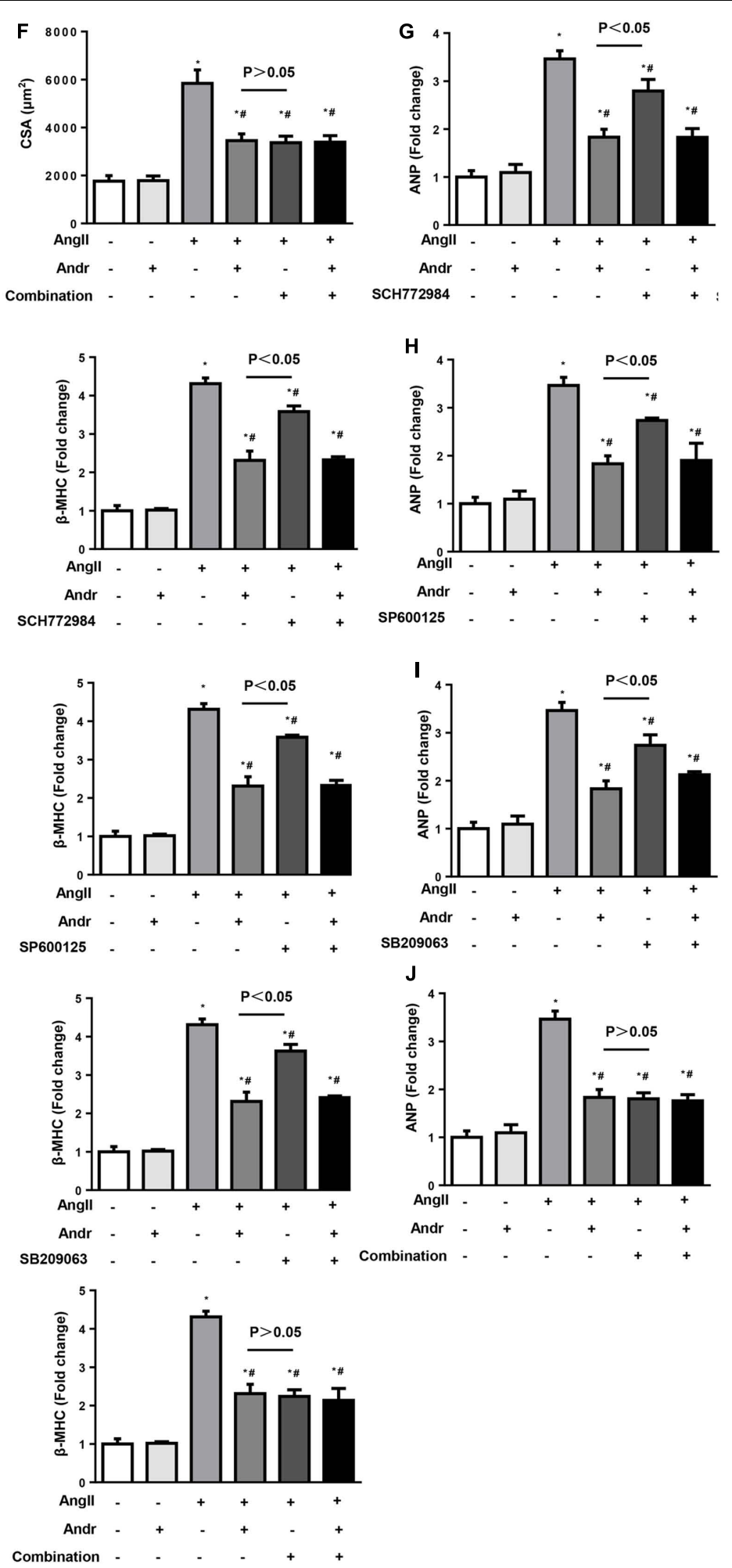

FIGURE 6 | Andr-mediated cardioprotection depends on the inhibition of MAPKs in cardiomyocytes. Cardiomyocytes were treated with ERK1/2 inhibitor (SCH7729, $5 \mu \mathrm{M})$, JNK inhibitor (SP600125, $10 \mu \mathrm{M})$, and/or P38 inhibitor (SB209063, $10 \mu \mathrm{M})$ as well as stimulated with Ang II (1 $\mu \mathrm{M})$ and treated with Andr (50 $\mu \mathrm{M})$. (A) Cell viability was accessed by the cell counting kit-8 assay $(n=5)$. (B-E) Immunofluorescence staining of $\alpha$-actinin and the cell surface area of cardiomyocytes in the indicated groups ( $n=5$ samples and $n=100+$ cells per group). (F-J) The mRNA levels of ANP and $\beta$-MHC in cardiomyocytes in the indicated groups ( $n=6)$. The results are presented as a fold change, and the results are normalized to GAPDH gene expression. ${ }^{*} P<0.05$ compared with the control group. ${ }^{\#} P<0.05$ vs. the Ang II group. 


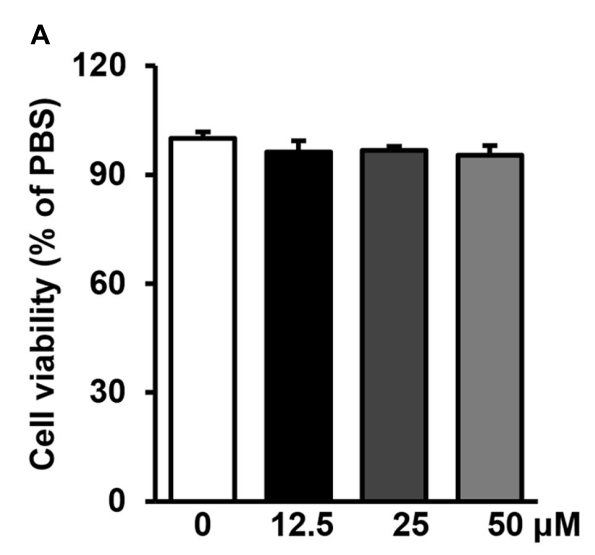

C

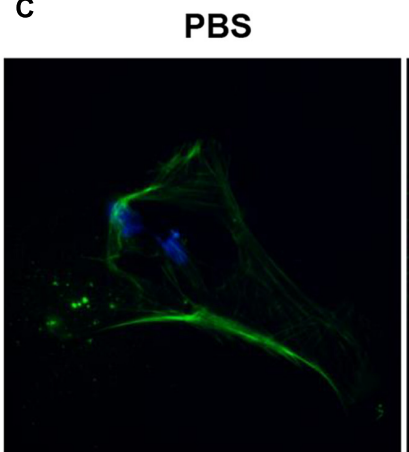

$25 \mu \mathrm{M}$
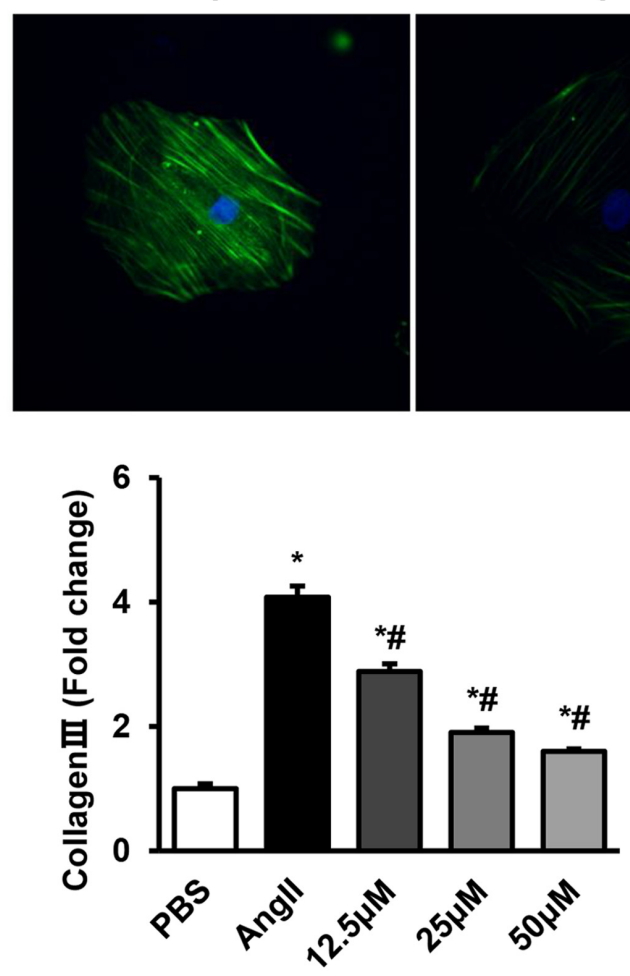

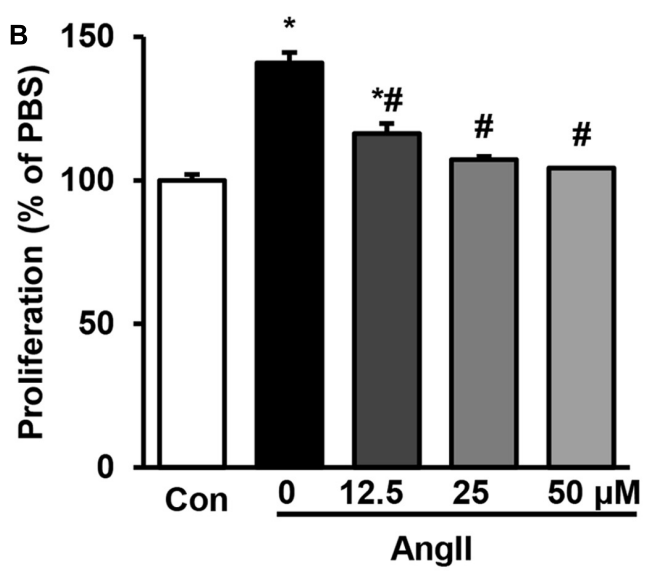

Angll

$12.5 \mu \mathrm{M}$

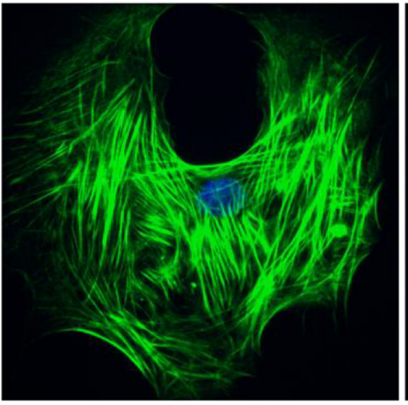

$50 \mu \mathrm{M}$
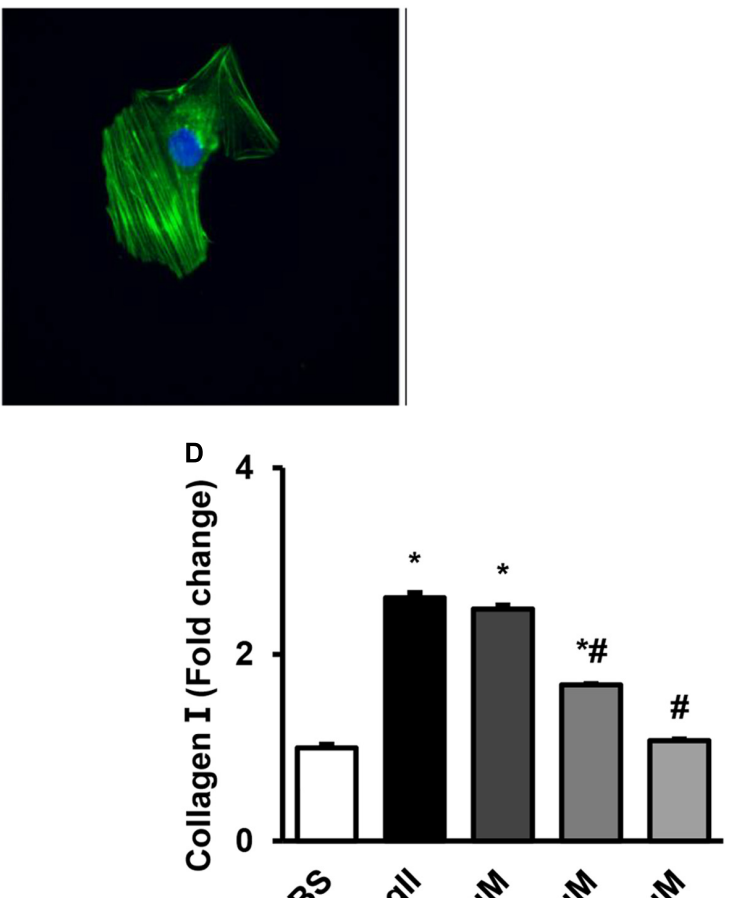

$50 \mu \mathrm{m}$

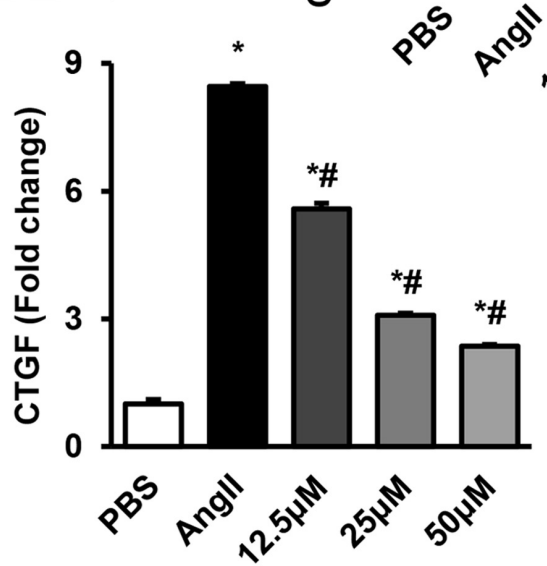



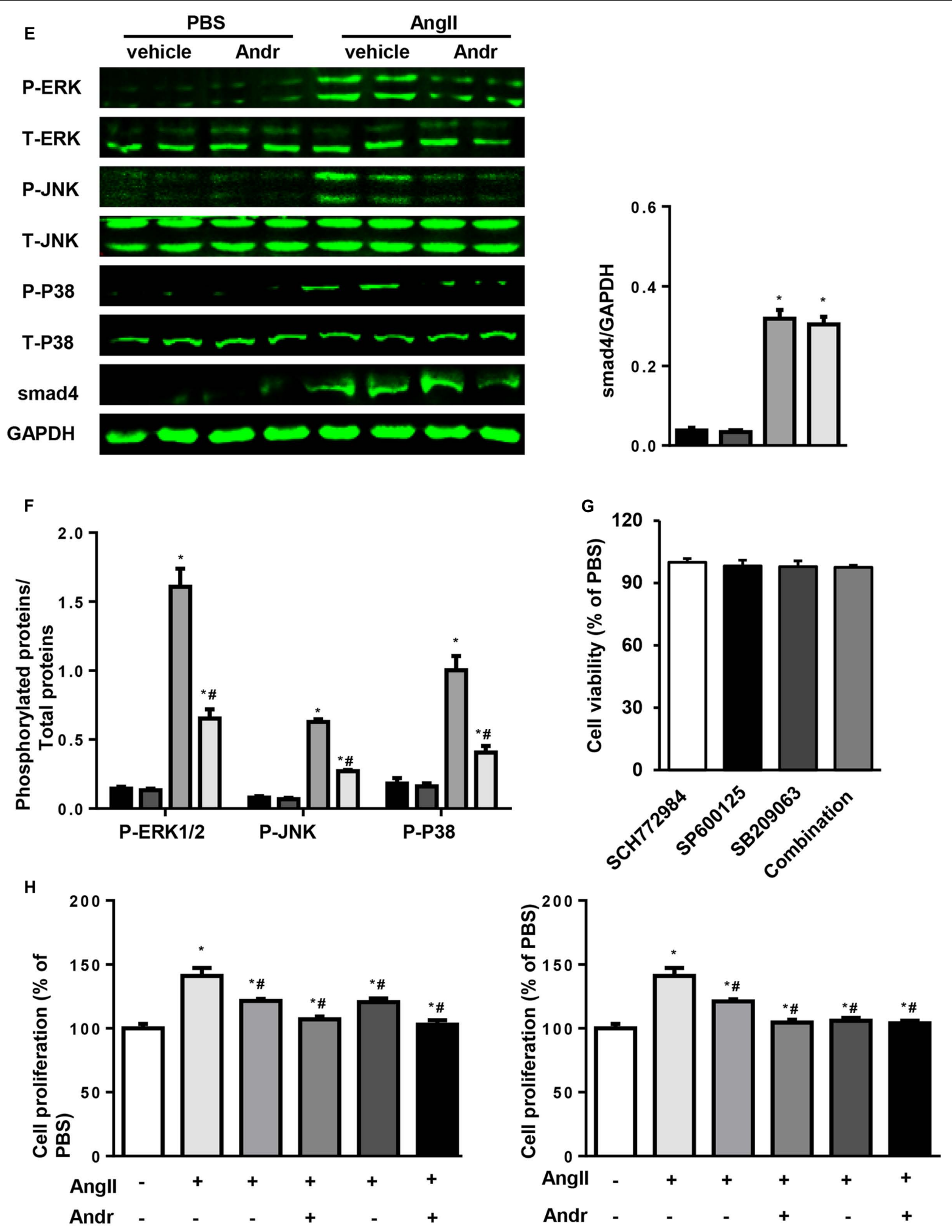

SCH772984 - $\quad+\quad+\quad+\quad-\quad+\quad$ SB209063

SP600125 

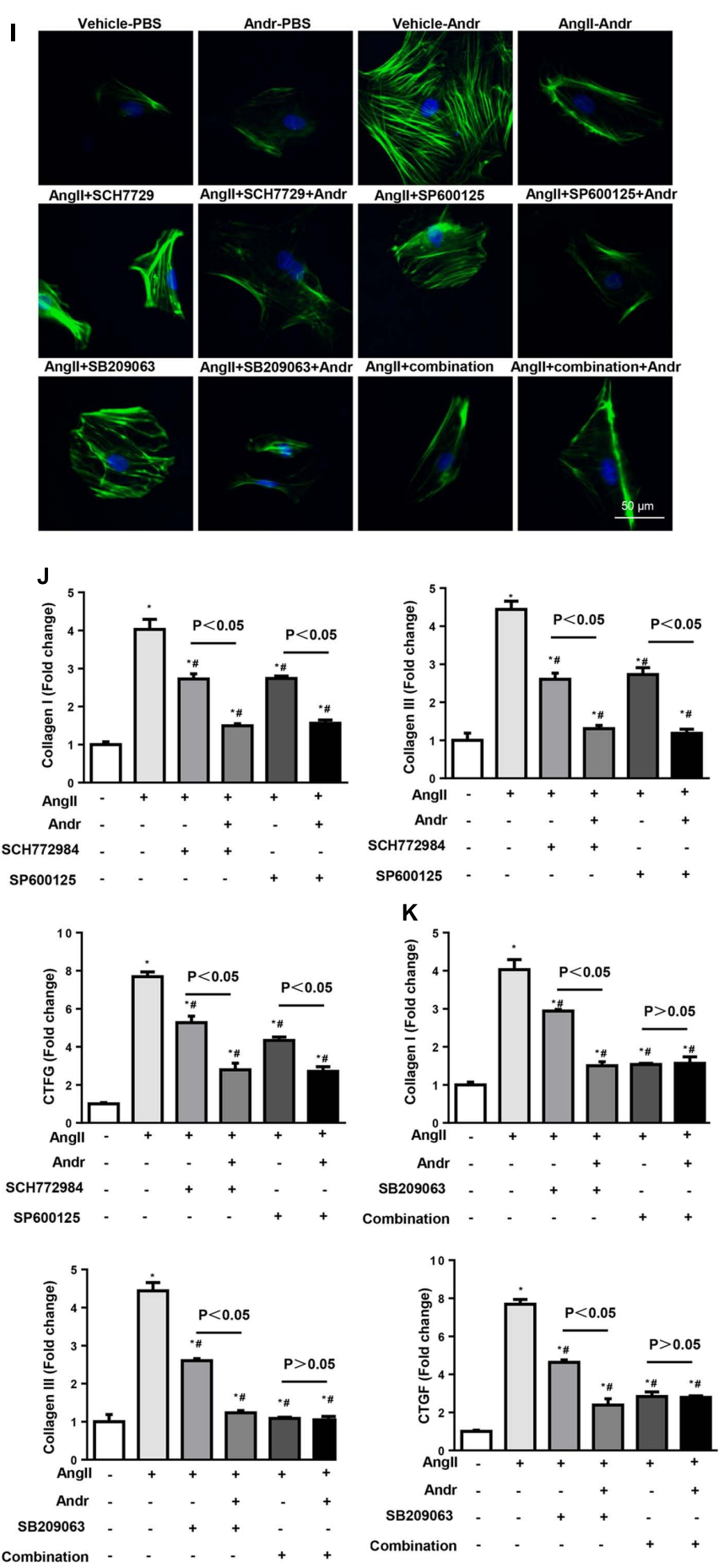

FIGURE 7 | Andr reduces cardiac fibroblast activation via MAPKs. (A-F) Cardiac fibroblasts were treated with different concentrations of Andr (0, 12.5, 25, or $50 \mu \mathrm{M})$ and/or stimulated with Ang II $(1 \mu \mathrm{M})$. (A) Cell viability was accessed by the cell counting kit-8 assay $(n=5)$. (B) Cell proliferation was accessed by the cell counting kit-8 assay $(n=5)$. (C) Immunofluorescence staining of $\alpha$-SMA in the indicated groups. (D) The mRNA levels of collagen I, collagen III, and CTGF in cardiac fibroblasts in the indicated groups $(n=6)$. (E) Representative blots of phosphorylated (P-) and total (T-) ERK1/2, JNK, P38, and smad4 in the cardiac fibroblasts in the indicated groups $(n=6)$. (F) Comparison of expression among the indicated groups. ${ }^{*} P<0.05$ compared with the corresponding PBS group. \#P $<0.05$ vs. the vehicle-Ang II group. (G-J) Cardiac fibroblasts were treated with ERK1/2 inhibitor (SCH7729, $5 \mu$ M), JNK inhibitor (SP600125, $10 \mu \mathrm{M})$, and/or P38 inhibitor (SB209063, $10 \mu \mathrm{M})$ as well as stimulated with Ang $\|(1 \mu \mathrm{M})$ and treated with Andr $(50 \mu \mathrm{M})$. (G) Cell viability was accessed by the cell counting kit-8 assay $(n=5)$. (H) Cell proliferation was accessed by the cell counting kit-8 assay $(n=5)$. (I) Immunofluorescence staining of $\alpha$-SMA in the indicated groups. (J,K) The mRNA levels of collagen I, collagen III, and CTGF in cardiac fibroblasts in the indicated groups $(n=6)$. The results are presented as a fold change, and the results are normalized to GAPDH gene expression. ${ }^{*} P<0.05$ compared with the control group. ${ }^{\#} P<0.05$ vs. the Ang $I I$ group. 
P38) in fibroblasts (Figures 7E,F), which was confirmed by ERK1/2 inhibitor, JNK inhibitor, and P38 inhibitor treatment (Figures 7H-K). However, Andr did not affect Ang II-induced smad4 expression (Figures 7E,F). Fibroblast viability was neither affected by single MAPKs inhibitor treatment nor treatment with a combination of MAPKs inhibitors (Figure 7G). These data suggest that the suppression of MAPKs in cardiac fibroblasts contributes to the anti-fibrosis effect of Andr on fibroblasts.

\section{DISCUSSION}

Pressure overload induces cardiomyocyte hypertrophy and fibroblast activation in cardiac tissue, resulting in cardiac hypertrophy and fibrosis followed by cardiac dysfunction in both human failure hearts (Gjesdal et al., 2011) and mouse models (Wu et al., 2015). Andr pharmacologically attenuated cardiac hypertrophy and fibrosis in vivo. In addition, our study also demonstrated that Andr ameliorated the Ang II-induced hypertrophic response in myocytes in vitro, as well as alleviated Ang II-induced fibroblast activation, proliferation, and function. Regarding the mechanism, we found that Andr suppressed the activation of ERK1/2, JNK, and P38 in both cardiomyocytes and fibroblasts ameliorating cardiac hypertrophy and fibrosis in the heart and improving cardiac function.

The mechanisms underlying the cardio-protective effects of Andr are not clear. In human patients with heart failure, MAPK signaling proteins have been reported to be hyperactive (Haque and Wang, 2017). Under physiological conditions MAPKs regulate cell proliferation and differentiation, whereas under pathological conditions, activated MAPKs induce the hypertrophic gene transcription (Liu and Molkentin, 2016). The downstream targets of MAPKs kinases include P38 kinase, JNK, and ERK1/2. Studies have shown that constitutive activation of ERK1/2 kinase contributes to concentric hypertrophy in cardiomyocytes (Kehat and Molkentin, 2010); ERK1/2 act upon nuclear factor of activated T-cells to mediate cardiac hypertrophy (Molkentin, 2013). JNK activation results in increased mitochondrion-associated apoptosis and fibrosis in the heart (Rose et al., 2010). Activation of JNK contributes to restrictive cardiomyopathy and promotes fibrosis in the heart (Petrich et al., 2004). P38 is also involved in myocyte growth. P38 inhibitors (SB203580 or SB202190) suppressed hypertrophic stimuli induced myocyte growth and dominant negative P38 delivered by adenoviruses (Zechner et al., 1997; Liang and Molkentin, 2003). Our previous studies have reported that many molecular and plant extracts ameliorate pressure overload-induced cardiac hypertrophy via MAPKs inhibition (Wu et al., 2017b). ATF3 exerts negative feedback on the ERK and JNK pathways to modulate cardiac remodeling (Zhou et al., 2011). Mnk1 prevents cardiac hypertrophy by inhibiting the Ras/ERK pathway (Yuan et al., 2016). Other plant-derived inhibitors, such as geniposide, indole-3-carbinol, and baicalein, also target on MAPKs signaling proteins (Wu et al., 2017b). These findings do not indicate whether a particular molecule is definitively 'bad' or 'good.' By focusing on network interactions rather than signal molecules, we may have a better chance at influencing the outcome. Previous reports have indicated that Andr exerts various effects by regulating MAPKs. Andr ameliorates rheumatoid arthritis by inhibiting MAPKs pathways (Li Z.Z. et al., 2017). Andr protects against ischemic stroke in rats by regulating the MAPKs signaling cascade (Yen et al., 2016). Andr reduces IL-2 production in T-cells by interfering with NFAT and ERK activation (Burgos et al., 2005; Carretta et al., 2009). Conversely, Andr induces Nrf2 and HO-1 in astrocytes by activating p38 and ERK (Wong et al., 2016). Andr inhibits the growth of human T-cell acute lymphoblastic leukemia Jurkat cells by upregulating of P38 pathways (Yang et al., 2016). These results indicate that Andr differentially regulates MAPKs in different cell types. Our results revealed that Andr inhibits the three terminal effectors of the MAPKs signaling cascade following induction by hypertrophic stimuli in both cardiomyocytes and fibroblasts. Using ERK1/2, JNK, and P38 inhibitors, we further demonstrated that Andr exerts cardio-protective effects by inhibiting both ERK, JNK, and P38. Andr exhibits similar inhibition efficiency for ERK, JNK, and P38. As our result in Figure 6 shows, single MAPK protein inhibition exerts similar anti-hypertrophic effect. Andr equally augmented these anti-hypertrophic responses. Direct evidence of the inhibition efficiency of Andr for ERK, JNK, and P38 requires further study.

Cardiac fibrosis is a major feature of hypertrophic cardiomyopathy and contributes to ventricular dysfunction and life-threatening arrhythmia (Travers et al., 2016). Our in vivo study showed that Andr treatment abated the pressure overloadinduced fibrotic response. Cardiac fibroblasts contribute to the heart's response to various forms of injury. After myocardial injury, the expression of various pro-fibrotic factors is up-regulated in fibroblasts, leading to increased fibroblast cell proliferation and ultimately, its transition to the myofibroblast phenotype (Kurose and Mangmool, 2016). Under these conditions, a subset of activated myofibroblasts acquire new phenotypic characteristics, including expression of the contractile protein $\alpha$-SMA, and contribute to pathological cardiac remodeling (Tomasek et al., 2002). Considering the key role of fibroblasts in cardiac fibrosis, we wondered whether Andr could directly target fibroblasts. Our in vitro results showed that Andr ameliorates Ang II-induced fibroblast activation, proliferation, and function I. The AT1R mediates many effects of Ang II in fibroblasts, including cell proliferation, cell migration, and the induction of extracellular matrix protein synthesis (Gao et al., 2009). AT1R activation results in the G-proteindependent activation of MAPKs, which leads to the activation and expression of collagen in fibroblasts (Dostal et al., 2015). Ang II is also involved in TGF $\beta /$ smad signaling. Activation of AT1R by Ang II induces the expression TGF- $\beta 1$ (Ma et al., 2012). We found that Andr suppressed the activation of MAPKs. MAPK inhibition by a combination of specific inhibitors exerted the same protective effects as Andr. These results indicate that the anti-fibrotic effect of Andr depends the inhibition of MAPKs. In contrast, Andr did not affect smad4 expression, indicating that smad signaling may not be a target of Andr.

In the in vivo study, $25 \mathrm{mg} / \mathrm{kg} /$ day Andr was used from 1 week after surgery to 8 weeks after surgery. A pharmacokinetic study reported that the blood concentration-time curve of Andr by 
oral gavage in rats $(10 \mathrm{mg} / \mathrm{kg})$ was fitted to the one-compartment model, in which the blood concentration of Andr increases sharply to $1.6 \mu \mathrm{g} / \mathrm{mL}$ in $100 \mathrm{~min}$ and gradually decreases within $600 \mathrm{~min}$ (Suo et al., 2007). The $25 \mathrm{mg} / \mathrm{kg} /$ day oral dose via gavage that was used in our study could maintain the blood Andr concentration at a certain level. However, accurate pharmacokinetic measurement in mice requires further study.

\section{CONCLUSION}

We documented the effective inhibition of the MAPKs signaling pathway by Andr treatment in both cardiomyocytes and fibroblasts and showed that MAPKs mediates the anti-hypertrophic effect of Andrin heart tissue. Cardiac hypertrophy due to stress, such as pressure overload, often culminates in heart failure and is associated with adverse cardiovascular events. Classical pharmacological treatment strategies for heart failure are ineffective in a number of patients. Although the effect of Andr on human cardiac hypertrophy and

\section{REFERENCES}

Banerjee, A., Banerjee, V., Czinn, S., and Blanchard, T. (2017). Increased reactive oxygen species levels cause ER stress and cytotoxicity in andrographolide treated colon cancer cells. Oncotarget 8, 26142-26153. doi: 10.18632/ oncotarget.15393

Burgos, R. A., Seguel, K., Perez, M., Meneses, A., Ortega, M., Guarda, M. I., et al. (2005). Andrographolide inhibits IFN-gamma and IL-2 cytokine production and protects against cell apoptosis. Planta Med. 71, 429-434. doi: 10.1055/s2005-864138

Carretta, M. D., Alarcon, P., Jara, E., Solis, L., Hancke, J. L., Concha, I. I., et al. (2009). Andrographolide reduces IL-2 production in T-cells by interfering with NFAT and MAPK activation. Eur. J. Pharmacol. 602, 413-421. doi: 10.1016/j. ejphar.2008.11.011

Chen, H. W., Huang, C. S., Li, C. C., Lin, A. H., Huang, Y. J., Wang, T. S., et al. (2014). Bioavailability of andrographolide and protection against carbon tetrachloride-induced oxidative damage in rats. Toxicol. Appl. Pharmacol. 280, 1-9. doi: 10.1016/j.taap.2014.07.024

Dostal, D., Glaser, S., and Baudino, T. A. (2015). Cardiac fibroblast physiology and pathology. Compr. Physiol. 5, 887-909. doi: 10.1002/cphy.c140053

Gao, X., He, X., Luo, B., Peng, L., Lin, J., and Zuo, Z. (2009). Angiotensin II increases collagen I expression via transforming growth factor-betal and extracellular signal-regulated kinase in cardiac fibroblasts. Eur. J. Pharmacol. 606, 115-120. doi: 10.1016/j.ejphar.2008.12.049

Gjesdal, O., Bluemke, D. A., and Lima, J. A. (2011). Cardiac remodeling at the population level-risk factors, screening, and outcomes. Nat. Rev. Cardiol. 8, 673-685. doi: 10.1038/nrcardio.2011.154

Haque, Z. K., and Wang, D. Z. (2017). How cardiomyocytes sense pathophysiological stresses for cardiac remodeling. Cell Mol. Life Sci. 74, 983-1000. doi: 10.1007/s00018-016-2373-0

Hill, J. A., and Olson, E. N. (2008). Cardiac plasticity. N. Engl. J. Med. 358, 1370-1380. doi: 10.1056/NEJMra072139

Hou, J., and Kang, Y. J. (2012). Regression of pathological cardiac hypertrophy: signaling pathways and therapeutic targets. Pharmacol. Ther. 135, 337-354. doi: $10.1016 /$ j.pharmthera.2012.06.006

Hsieh, Y. L., Shibu, M. A., Lii, C. K., Viswanadha, V. P., Lin, Y. L., Lai, C. H., et al. (2016). Andrographis paniculata extract attenuates pathological cardiac hypertrophy and apoptosis in high-fat diet fed mice. J. Ethnopharmacol. 192, 170-177. doi: 10.1016/j.jep.2016.07.018

Kehat, I., and Molkentin, J. D. (2010). Extracellular signal-regulated kinase 1/2 (ERK1/2) signaling in cardiac hypertrophy. Ann. N. Y. Acad. Sci. 1188, 96-102. doi: $10.1111 /$ j.1749-6632.2009.05088.x heart failure has not yet been reported, these observations in mice are critical for the development of treatment strategies for cardiac hypertrophy and heart failure.

\section{AUTHOR CONTRIBUTIONS}

QW, QT, and WD contributed to the conception, and design of the experiments; QW, JN, and WD carried out the experiments; $\mathrm{NZ}$ and HL analyzed the experimental results, and revised the manuscript. QW and WD wrote and revised the manuscript.

\section{FUNDING}

This work was supported by grants from the National Natural Science Foundation of China (Nos. 81470516, 81530012, and 81700353), Hubei Province's Outstanding Medical Academic Leader Program, and the Fundamental Research Funds of the Central Universities (2042017kf0060).

Kurose, H., and Mangmool, S. (2016). Myofibroblasts and inflammatory cells as players of cardiac fibrosis. Arch. Pharm. Res. 39, 1100-1113. doi: 10.1007/ s12272-016-0809-6

Li, W. M., Zhao, Y. F., Zhu, G. F., Peng, W. H., Zhu, M. Y., Yu, X. J., et al. (2017). Dual specific phosphatase 12 ameliorates cardiac hypertrophy in response to pressure overload. Clin. Sci. 131, 141-154. doi: 10.1042/CS201 60664

Li, Y., Yan, H., Zhang, Z., Zhang, G., Sun, Y., Yu, P., et al. (2015). Andrographolide derivative AL-1 improves insulin resistance through down-regulation of NFКB signalling pathway. Br. J. Pharmacol. 172, 3151-3158. doi: 10.1111/bph. 13118

Li, Z. Z., Tan, J. P., Wang, L. L., and Li, Q. H. (2017). Andrographolide benefits rheumatoid arthritis via inhibiting MAPK pathways. Inflammation 40, 1599-1605. doi: 10.1007/s10753-017-0600-y

Liang, Q., and Molkentin, J. D. (2003). Redefining the roles of p38 and JNK signaling in cardiac hypertrophy: dichotomy between cultured myocytes and animal models. J. Mol. Cell Cardiol. 35, 1385-1394. doi: 10.1016/j.yjmcc.2003. 10.001

Liao, P., Georgakopoulos, D., Kovacs, A., Zheng, M., Lerner, D., Pu, H., et al. (2001). The in vivo role of p38 MAP kinases in cardiac remodeling and restrictive cardiomyopathy. Proc. Natl. Acad. Sci. U.S.A. 98, 12283-12288. doi: 10.1073/ pnas. 211086598

Liu, R., and Molkentin, J. D. (2016). Regulation of cardiac hypertrophy and remodeling through the dual-specificity MAPK phosphatases (DUSPs). J. Mol. Cell Cardiol. 101, 44-49. doi: 10.1016/j.yjmcc.2016.08.018

Lorenz, K., Schmitt, J. P., Schmitteckert, E. M., and Lohse, M. J. (2009). A new type of ERK1/2 autophosphorylation causes cardiac hypertrophy. Nat. Med. 15, 75-83. doi: 10.1038/nm.1893

Lyon, R. C., Zanella, F., Omens, J. H., and Sheikh, F. (2015). Mechanotransduction in cardiac hypertrophy and failure. Circ. Res. 116, 1462-1476. doi: 10.1161/ CIRCRESAHA.116.304937

Ma, F., Li, Y., Jia, L., Han, Y., Cheng, J., Li, H., et al. (2012). Macrophagestimulated cardiac fibroblast production of IL-6 is essential for TGF $\beta / \mathrm{Smad}$ activation and cardiac fibrosis induced by angiotensin II. PLOS ONE 7:e35144. doi: 10.1371/journal.pone.0035144

Martin, T. P., Robinson, E., Harvey, A. P., MacDonald, M., Grieve, D. J., Paul, A., et al. (2012). Surgical optimization and characterization of a minimally invasive aortic banding procedure to induce cardiac hypertrophy in mice. Exp. Physiol. 97, 822-832. doi: 10.1113/expphysiol.2012.065573

Molkentin, J. D. (2013). Parsing good versus bad signaling pathways in the heart: role of calcineurin-nuclear factor of activated T-cells. Circ. Res. 113, 16-19. doi: 10.1161/CIRCRESAHA.113.301667 
Pan, C. W., Yang, S. X., Pan, Z. Z., Zheng, B., Wang, J. Z., Lu, G. R., et al. (2017). Andrographolide ameliorates d-galactosamine/lipopolysaccharideinduced acute liver injury by activating Nrf2 signaling pathway. Oncotarget 8 , 41202-41210. doi: 10.18632/oncotarget.17149

Peng, S., Hang, N., Liu, W., Guo, W., Jiang, C., Yang, X., et al. (2016). Andrographolide sulfonate ameliorates lipopolysaccharide-induced acute lung injury in mice by down-regulating MAPK and NF-kappaB pathways. Acta Pharm. Sin. B 6, 205-211. doi: 10.1016/j.apsb.2016. 02.002

Petrich, B. G., Eloff, B. C., Lerner, D. L., Kovacs, A., Saffitz, J. E., Rosenbaum, D. S., et al. (2004). Targeted activation of c-Jun N-terminal kinase in vivo induces restrictive cardiomyopathy and conduction defects. J. Biol. Chem. 279, 15330-15338. doi: 10.1074/jbc.M314142200

Ren, J., Liu, Z., Wang, Q., Giles, J., Greenberg, J., Sheibani, N., et al. (2016). Andrographolide ameliorates abdominal aortic aneurysm progression by inhibiting inflammatory cell infiltration through downregulation of cytokine and integrin expression. J. Pharmacol. Exp. Ther. 356, 137-147. doi: 10.1124/ jpet.115.227934

Rose, B. A., Force, T., and Wang, Y. (2010). Mitogen-activated protein kinase signaling in the heart: angels versus demons in a heart-breaking tale. Physiol. Rev. 90, 1507-1546. doi: 10.1152/physrev.00054.2009

Samak, M., Fatullayev, J., Sabashnikov, A., Zeriouh, M., Schmack, B., Farag, M., et al. (2016). Cardiac hypertrophy: an introduction to molecular and cellular basis. Med. Sci. Monit. Basic Res. 22, 75-79. doi: 10.12659/MSMBR. 900437

Shimizu, I., and Minamino, T. (2016). Physiological and pathological cardiac hypertrophy. J. Mol. Cell Cardiol. 97, 245-262. doi: 10.1016/j.yjmcc.2016.06.001

Suo, X. B., Zhang, H., and Wang, Y. Q. (2007). HPLC determination of andrographolide in rat whole blood: study on the pharmacokinetics of andrographolide incorporated in liposomes and tablets. Biomed. Chromatogr. 21, 730-734. doi: 10.1002/bmc.812

Tham, Y. K., Bernardo, B., Ooi, J. Y., Weeks, K. L., and McMullen, J. R. (2015). Pathophysiology of cardiac hypertrophy and heart failure: signaling pathways and novel therapeutic targets. Arch. Toxicol. 89, 1401-1438. doi: 10.1007/ s00204-015-1477-x

Tomasek, J. J., Gabbiani, G., Hinz, B., Chaponnier, C., and Brown, R. A. (2002). Myofibroblasts and mechano-regulation of connective tissue remodelling. Nat. Rev. Mol. Cell Biol. 3, 349-363. doi: 10.1038/nrm809

Travers, J. G., Kamal, F., Robbins, J., Yutzey, K. E., and Blaxall, B. C. (2016). Cardiac fibrosis: the fibroblast awakens. Circ. Res. 118, 1021-1040. doi: 10.1161/ CIRCRESAHA.115.306565

Wong, S. Y., Tan, M. G., Wong, P. T., Herr, D. R., and Lai, M. K. (2016). Andrographolide induces Nrf2 and heme oxygenase 1 in astrocytes by activating p38 MAPK and ERK. J. Neuroinflammation 13, 251. doi: 10.1186/ s12974-016-0723-3

Woo, A. Y., Waye, M. M., Tsui, S. K., Yeung, S. T., and Cheng, C. H. (2008). Andrographolide up-regulates cellular-reduced glutathione level and protects cardiomyocytes against hypoxia/reoxygenation injury. J. Pharmacol. Exp. Ther. 325, 226-235. doi: 10.1124/jpet.107.133918

Wu, Q. Q., Xiao, Y., Jiang, X. H., Yuan, Y., Yang, Z., Chang, W., et al. (2017a). Evodiamine attenuates TGF-betal-induced fibroblast activation and endothelial to mesenchymal transition. Mol. Cell. Biochem. 430, 81-90. doi: $10.1007 /$ s11010-017-2956-6
Wu, Q. Q., Xiao, Y., Yuan, Y., Ma, Z. G., Liao, H. H., Liu, C., et al. (2017b). Mechanisms contributing to cardiac remodelling. Clin. Sci. 131, 2319-2345. doi: 10.1042/CS20171167

Wu, Q. Q., Xu, M., Yuan, Y., Li, F. F., Yang, Z., Liu, Y., et al. (2015). Cathepsin $\mathrm{B}$ deficiency attenuates cardiac remodeling in response to pressure overload via TNF-alpha/ASK1/JNK pathway. Am. J. Physiol. Heart Circ. Physiol. 308, H1143-H1154. doi: 10.1152/ajpheart.00601.2014

Wu, Q. Q., Yuan, Y., Jiang, X. H., Xiao, Y., Yang, Z., Ma, Z. G., et al. (2016). OX40 regulates pressure overload-induced cardiac hypertrophy and remodelling via CD4 ${ }^{+}$T-cells. Clin. Sci. 130, 2061-2071. doi: 10.1042/CS20160074

Yang, R., Liu, S., Zhou, J., Bu, S., and Zhang, J. (2017). Andrographolide attenuates microglia-mediated $A \beta$ neurotoxicity partially through inhibiting NF- $\mathrm{B}$ and JNK MAPK signaling pathway. Immunopharmacol. Immunotoxicol. 39, 276-284. doi: 10.1080/08923973.2017.1344989

Yang, T., Yao, S., Zhang, X., and Guo, Y. (2016). Andrographolide inhibits growth of human T-cell acute lymphoblastic leukemia Jurkat cells by downregulation of PI3K/AKT and upregulation of p38 MAPK pathways. Drug Des. Dev. Ther. 10, 1389-1397. doi: 10.2147/DDDT.S94983

Yen, T. L., Chen, R. J., Jayakumar, T., Lu, W. J., Hsieh, C. Y., Hsu, M. J., et al. (2016). Andrographolide stimulates p38 mitogen-activated protein kinasenuclear factor erythroid-2-related factor 2-heme oxygenase 1 signaling in primary cerebral endothelial cells for definite protection against ischemic stroke in rats. Transl. Res. 170, 57-72. doi: 10.1016/j.trsl.2015.12.002

Yuan, Y., Yan, L., Wu, Q. Q., Zhou, H., Jin, Y. G., Bian, Z. Y., et al. (2016). Mnk1 (mitogen-activated protein kinase-interacting kinase 1) deficiency aggravates cardiac remodeling in mice. Hypertension 68, 1393-1399. doi: 10.1161/ HYPERTENSIONAHA.116.07906

Zechner, D., Thuerauf, D. J., Hanford, D. S., McDonough, P. M., and Glembotski, C. C. (1997). A role for the p38 mitogen-activated protein kinase pathway in myocardial cell growth, sarcomeric organization, and cardiac-specific gene expression. J. Cell Biol. 139, 115-127. doi: 10.1083/jcb.139.1.115

Zeng, M., Jiang, W., Tian, Y., Hao, J., Cao, Z., Liu, Z., et al. (2017). Andrographolide inhibits arrhythmias and is cardioprotective in rabbits. Oncotarget 8, 61226-61238. doi: 10.18632/oncotarget.18051

Zhang, J., Zhu, D., Wang, Y., and Ju, Y. (2015). Andrographolide attenuates LPS-induced cardiac malfunctions through inhibition of IкB phosphorylation and apoptosis in mice. Cell Physiol. Biochem. 37, 1619-1628. doi: 10.1159/ 000438528

Zhou, H., Shen, D. F., Bian, Z. Y., Zong, J., Deng, W., Zhang, Y., et al. (2011). Activating transcription factor 3 deficiency promotes cardiac hypertrophy, dysfunction, and fibrosis induced by pressure overload. PLOS ONE 6:e26744. doi: 10.1371/journal.pone.0026744

Conflict of Interest Statement: The authors declare that the research was conducted in the absence of any commercial or financial relationships that could be construed as a potential conflict of interest.

Copyright (c) $2017 \mathrm{Wu}, \mathrm{Ni}$, Zhang, Liao, Tang and Deng. This is an open-access article distributed under the terms of the Creative Commons Attribution License (CC BY). The use, distribution or reproduction in other forums is permitted, provided the original author(s) or licensor are credited and that the original publication in this journal is cited, in accordance with accepted academic practice. No use, distribution or reproduction is permitted which does not comply with these terms. 